TRANSACTIONS OF THE

AMERICAN MATHEMATICAL SOCIETY

Volume 350, Number 2, February 1998, Pages 809-849

S 0002-9947(98)02107-2

\title{
RECOGNIZING CONSTANT CURVATURE DISCRETE GROUPS IN DIMENSION 3
}

\author{
J. W. CANNON AND E. L. SWENSON
}

\begin{abstract}
We characterize those discrete groups $G$ which can act properly discontinuously, isometrically, and cocompactly on hyperbolic 3-space $\mathbb{H}^{3}$ in terms of the combinatorics of the action of $G$ on its space at infinity. The major ingredients in the proof are the properties of groups that are negatively curved (in the large) (that is, Gromov hyperbolic), the combinatorial Riemann mapping theorem, and the Sullivan-Tukia theorem on groups which act uniformly quasiconformally on the 2-sphere.
\end{abstract}

\section{INTRODUCTION}

Characteristic features of hyperbolic non-Euclidean space $\mathbb{H}^{n}$ are its negative curvature and its spherical space $\mathbf{S}^{n-1}$ at infinity. A finitely-generated group $G$ which acts properly discontinuously, isometrically, and cocompactly on $\mathbb{H}^{n}$ captures the geometry of $\mathbb{H}^{n}$ and its space $\mathbf{S}^{n-1}$ at infinity in three interesting ways: (1) the negative curvature of $\mathbb{H}^{n}$ is realized in the group $G$ as negative curvature (in the large) of the Cayley graph $\Gamma$ of $G$ (also known as Gromov word hyperbolicity [15]); (2) this negative curvature ensures that the combinatorics and asymptotic behavior of the group are computationally simple as captured by the notion of automatic group [11]; and (3) the topology of the spherical space at infinity can be extracted from the asymptotic behavior of the group itself [15].

Our aim is to characterize such groups, at least in dimension 3 , in terms of their combinatorial asymptotic behavior at infinity.

The principal difficulty we address is this: how does one distinguish a group acting on a space of constant negative curvature from one acting on a space of variable negative curvature? In high dimensions, the groups associated with constant curvature form a proper subclass of those associated with variable negative curvature. (See, for example, [23] and [16]. Compare with [20] and [29].) In dimension 2 the two classes coincide. In dimension 3 the two classes may coincide, as required by Thurston's as yet unresolved geometrization conjecture [28]. Our hope is that our result may prove useful in resolving that problem.

We now review the asymptotic features in terms of which we intend to state our characterization theorem.

Received by the editors July 13, 1994 and, in revised form, November 14, 1996.

1991 Mathematics Subject Classification. Primary 20F32, 30F40, 57N10; Secondary 30C62, $31 \mathrm{~A} 15,30 \mathrm{~F} 10$.

This research was supported in part by The Geometry Center at the University of Minnesota, a Science and Technology Center funded by NSF, DOE, and Minnesota Technology, Inc.; and by NSF Research Grant No. DM-8902071.

(C) 1998 American Mathematical Society 
1.1. Cayley graphs. A group $G$ with finite generating set $C=C^{-1}$ acts properly discontinuously, isometrically, and cocompactly on its Cayley graph $\Gamma=\Gamma(G, C)$. (See, for example, [5], [6].) The graph $\Gamma$ forms a combinatorial substitute for $\mathbb{H}^{n}$.

1.2. Negative curvature in the large, and thin polygons. A metric space is proper if its closed metric balls are compact. A space $X$ with proper path metric is negatively curved (in the large) (also known as Gromov hyperbolic [15]) if its triangles are uniformly thin. That is, there is a non-negative constant $\delta$ such that any point of one side of the triangle lies within $\delta$ of one of the other two sides. Gromov hyperbolic groups are studied, for example, in [1], [2], [4], [6], [10], [15], [27].

It is an easy exercise to show that if triangles are $\delta$-thin, then $n$-gons are $(n-2) \delta$ thin for all $n>3$. Trees are negatively curved with thinness constant 0 . Hyperbolic space is negatively curved with thinness constant $\delta=\log (1+\sqrt{2})$ (see [6]). Negative curvature of the Cayley graph is an invariant of the group [1], [2], [4], [6], [10], [27] in the sense that, if it is satisfied for one locally finite Cayley graph of $G$, it is satisfied, with possibly different thinness constant, by every other locally finite Cayley graph of $G$. Thus a group $G$ is negatively curved if every locally finite Cayley graph of $G$ is negatively curved. Negative curvature of $G$ captures the behavior in the large of a classical geometry having (possibly varying) negative curvature.

1.3. The space at infinity. A space $X$ with negatively curved proper path metric $d$ has a natural space at infinity, denoted $\partial X$. Points at infinity are equivalence classes $R(\infty)$ of rays $R:[0, \infty) \rightarrow X$, rays $R$ and $S$ being equivalent if $\lim \sup _{t \rightarrow \infty} d(R(t), S(t))<\infty$ or equivalently if the set $\{d(S(t), R)\}$ is bounded. The topology at infinity is defined in terms of (combinatorial) half spaces in $X$ and (combinatorial) disks at infinity in $\partial X$. Let $R:[a, b] \rightarrow X$ be a segment (possibly infinite) and $t \in[a, b]$ a (finite) number. The half-space $H(R, t)$ is defined by the formula

$$
H(R, t)=\{x \in X \mid d(x, R([t, b])) \leq d(x, R([a, t]))\}
$$

and the disk $D(R, t)$ by the formula

$$
D(R, t)=\left\{\left[R^{\prime}\right] \in \partial X \mid \lim _{r \rightarrow \infty} d\left(R^{\prime}(r), X \backslash H(R, t)\right)=\infty\right\} .
$$

The disks $D(R, t)$ form a basis for a compact metric topology on the set $\partial X$ (see [27]). The resulting space is homeomorphic with the ones defined by Furstenberg [13], Floyd [12], Gromov [15], and others. We are, however, particularly interested in the geometric and combinatorial behavior of the basis of disks $D(R, t)$. In the classical negatively-curved geometry of $\mathbb{H}^{n}$, the half-spaces are the standard classical geometric half-spaces and the disks at infinity are classical standard Euclidean open $(n-1)$-balls in the $(n-1)$-sphere $\mathbf{S}^{n-1}$ at infinity.

1.4. The characterization in outline. Our characterization will have three features: we require (1) that $\Gamma$ be negatively curved, (2) that the space $\partial \Gamma$ be the 2 -sphere, and (3) that the disks $D(R, t)$ cover $\partial \Gamma=\mathbf{S}^{2}$ in a combinatorially nice way. Our conclusion states that such conditions are necessary and sufficient in order that a group $G$ act properly discontinuously, isometrically, and cocompactly on $\mathbb{H}^{3}$. We save the precise definition of condition (3) for Section 2 of the paper. 
The chief technical apparatus for the proof is developed in two papers, [7] and [27]. The former has appeared in Acta Mathematica, but we include the necessary proofs from the latter.

We express thanks to a number of people who have contributed directly or indirectly to this work - Matt Grayson, who helped formulate an early version of condition (3); M. Gromov, who gave the general definition of negative curvature for discrete groups [15]; W. Parry and W. Floyd, who helped develop properties of combinatorial moduli [9]; W. Thurston, C. McMullen, P. Doyle, R. Schwarz, and O. Schramm, who discussed with us the physical and geometric significance of condition (3); M. Bestvina, who showed us an important averaging trick; B. Rodin, K. Stephenson, and A. Beardon, who discussed the relationship with circle packings and classical complex variables; A. Marden, who extended the hospitality of the Geometry Center; Ghys, de la Harpe, and others with whom we discussed the properties of negatively curved groups; D. Sullivan and Tukia, who supplied us with relevant papers on quasiconformal mappings; and many others.

\section{Statement of the main theorem}

Our goal is to recognize a cocompact discrete Kleinian group combinatorially. We are not trying just to approximate this structure, but rather to find the structure exactly. Our characterization is technical. Therefore we shall spend a fair amount of time in this exposition motivating the statement of the theorem and explaining the geometric and combinatorial implications of the theorem. We need to recall some history:

\subsection{History.}

Conjecture 2.1.1 [28]. A closed 3-manifold $M^{3}$ which admits a Riemannian metric of varying negative curvature also admits one of constant negative curvature.

If the conjecture is true, then the universal cover is isometric with hyperbolic 3 -space $\mathbb{H}^{3}$, and the fundamental group of $M^{3}$ acts geometrically on $\mathbb{H}^{3}$ and acts conformally on the 2 -sphere $\mathbf{S}^{2}$ at infinity.

Theorem 2.1.2 ([26] and [30]). In order that a group $G$ act geometrically on $\mathbb{H}^{3}$, it is necessary and sufficient that $G$ act discretely and uniformly quasiconformally on the 2-sphere $\mathbf{S}^{2}$.

2.2. Plan. In order to prove that a group acts uniformly quasiconformally on the 2 -sphere $\mathbf{S}^{2}$, we need a candidate action. We find that candidate in Gromov's theory [15] of negatively curved groups. (Recall the definitions from Section 1.) In order that a group act geometrically on $\mathbb{H}^{3}$, it has long been known that the group must be negatively curved in the sense of Gromov and that its natural space at infinity must be the 2 -sphere. (See, for example, [8].) The beginning point of our characterization is the assumption that $G$ is a negatively curved group with a 2-sphere as its space at infinity.

At this point the real difficulties of the paper begin. The space at infinity has only a topological structure and no obvious preferred smooth structure associated with the given group action. Gromov [15] has defined a metric at infinity on which the action is nicely behaved (see [10]), but it is likely that the 2-sphere with this metric is not smooth and in fact has Hausdorff dimension higher than the desired 
dimension 2. There are examples of semigroup actions on the plane which are uniformly quasiconformal in the sense of Gromov's metric which cannot be realized as uniformly quasiconformal in any conformal structure on the plane. (See Section 8.) Furthermore, among all possible topological conjugations of the action yielding a round 2-sphere as underlying space, we could use Mostow's rigidity theorem [22] and the Sullivan-Tukia theorem [26] [30] to show that there is at most one possible conjugation, up to quasiconformal homeomorphism, which makes the action uniformly quasiconformal in the classical sense. Among the uncountably many possible quasiconformal structures on $\mathbf{S}^{2}$, therefore, we are required to find exactly the right one.

2.2.1. Quasiconformal homeomorphisms. A homeomorphism of a spherical or planar domain is quasiconformal if, in the small, it distorts roundness a bounded amount. That is, there is a constant $K>0$ such that, if $C$ is a sufficiently small circle in the domain of the homeomorphism, then the image of $C$ separates an annulus having circle boundaries of radius $r_{1}$ and $r_{0}$ with $1<r_{1} / r_{0}<K$.

2.2.2. Almost round sets. Thus, in order to characterize a quasiconformal structure on a topological 2-sphere, it is necessary to decide which subsets of the 2 -sphere are, or should be, within bounded distortion of round, or, as we shall say, which subsets are almost round.

The sets which are almost round with respect to the standard structure on $\mathbf{S}^{2}$ may be defined as follows. Pick some distortion constant $K>0$. Let $X$ denote some subset of the 2 -sphere, and let $p$ be a point of $X$. Define the inner radius $r_{0}$ of $X$ with respect to $p$ to be the distance from $p$ to the complement of $X$. Define the outer radius $r_{1}$ of $X$ with respect to $p$ to be the supremum of the distances from $p$ to the points of $X$. We say that $X$ is almost round $(K)$ if there is a point $p \in X$ such that $\left(r_{1} / r_{0}\right)<K$.

2.2.3. Our tasks. Our tasks are (1) to pick out, using the combinatorial structure of the group $G$ alone, subsets of the 2 -sphere which are distorted a bounded amount by the group as measured by some unknown conformal structure on the surface and should be the class of almost round sets, (2) to show the existence of analytic coordinates on our topological 2-sphere under which the class of sets in question does indeed form the class of almost round sets, and (3) to show finally by means of the given sets that the group acts uniformly quasiconformally. The Sullivan-Tukia theorem then completes the characterization.

2.2.4. Facing the tasks. We discuss the following questions.

1. How are we to pick out the potentially almost round sets?

2. How should one measure potential roundness of a set in a combinatorial setting?

The combinatorial disks $D(R, t)$ defined in Section 1 will serve as our potential class of almost round sets. However, it is most unlikely that any given metric on our space at infinity will both make the space smooth and make the combinatorial disks round. We shall surely have to change our metric at least conformally, probably by a general homeomorphism. In view of this necessary change of coordinates, does it make sense to ask whether an individual set is almost round? Every individual topological disk is round up to homeomorphism by the Schoenflies theorem. Its interior is conformally round, by the Riemann mapping 
theorem, if one does not require that the homeomorphism act conformally on the entire 2-sphere. Thus it would seem that every topological disk should be almost round. But making one combinatorial disk metrically round may distort another combinatorial disk. The difficulty involves not individual disks but the whole family of disks taken together. Can they all together be made compatibly almost round? Our procedure will be to use the combinatorial disks to measure the roundness of all sets and to ask whether the information thus obtained is self-compatible.

The potential shape of a ring or annulus $\mathcal{R}$ under a conformal mapping of a spherical or planar domain is measured by a number that is called its conformal modulus. (See [19].) In electrical theory, these moduli measure the resistance of the ring $\mathcal{R}$ considered as a metallic conducting plate. In geometry, these moduli can be used to give a measure of the intrinsic roundness of $\mathcal{R}$. These moduli can be fairly well measured directly without actually finding a conformal map which takes the rings onto round annuli. We shall act as if the combinatorial disks were already almost round and defined a combinatorial metric on the 2 -sphere. We shall then change this metric in a combinatorially conformal way and optimize. The procedure is a combinatorial version of the classical length-area estimates of classical complex variable theory.

2.2.5. Combinatorial moduli and associated notions. Let $\mathcal{C}$ denote a finite covering of a topological annulus or ring $\mathcal{R}$. Let $\rho: \mathcal{C} \rightarrow[0, \infty)$ be an arbitrary assignment of nonnegative weights to the elements of $\mathcal{C}$. Then the $\rho$-area $A_{\rho}(\mathcal{R})$ of $\mathcal{R}$ is defined to be the sum of the squares of the weights of the elements of $\mathcal{C}$ that intersect $\mathcal{R}$. If $\alpha$ is an arc in $\mathcal{R}$, then the $\rho$-length $L_{\rho}(\alpha)$ of $\alpha$ is defined to be the sum of the weights of the elements of $\mathcal{C}$ that intersect $\alpha$. The $\rho$-height $H_{\rho}(\mathcal{R})$ of $\mathcal{R}$ is defined to be the minimum $\rho$-length of a curve in $\mathcal{R}$ joining the ends of $\mathcal{R}$. The $\rho$-circumference $C_{\rho}(\mathcal{R})$ of $\mathcal{R}$ is defined to be the minimum length of simple closed curves in $\mathcal{R}$ which circle the hole in $\mathcal{R}$. We then have the fundamental moduli

$$
M(\mathcal{R}, \mathcal{C})=\sup _{\rho}\left\{H_{\rho}(\mathcal{R})^{2} / A_{\rho}(\mathcal{R}) \mid A_{\rho}(\mathcal{R}) \neq 0\right\}
$$

and

$$
m(\mathcal{R}, \mathcal{C})=\inf _{\rho}\left\{A_{\rho}(\mathcal{R}) / C_{\rho}(\mathcal{R})^{2} \mid A_{\rho}(\mathcal{R}) \neq 0\right\} .
$$

A sequence $\mathcal{C}_{1}, \mathcal{C}_{2}, \ldots$ of finite covers of the 2 -sphere $\mathbf{S}^{2}$ or of the complex plane $\mathbb{C}$ is said to be conformal if the diameters of the elements go to zero locally uniformly as the index approaches infinity, and the following conditions are satisfied:

There is a positive number $K$ with the following properties:

1. Approximate moduli are approximately well-defined. That is, for each ring $\mathcal{R}$ in $\mathbf{S}^{2}$ (or $\mathbb{C}$ ), there is a positive number $m$, called an approximate modulus for $\mathcal{R}$, such that, for all $n$ sufficiently large, the two numbers $M_{n}(\mathcal{R})=$ $M\left(\mathcal{R}, \mathcal{C}_{n}\right)$ and $m_{n}(\mathcal{R})=m\left(\mathcal{R}, \mathcal{C}_{n}\right)$ lie in the interval $[m, K \cdot m]$.

2. Points are encircled by rings of arbitrarily large approximate modulus. That is, for each point $p \in \mathbf{S}^{2}(\mathbb{C})$, each neighborhood $N$ of $p$, and each positive number $P$, there is a ring $\mathcal{R}$ in $N$ separating $p$ from the complement of $N$ which has an approximate modulus $>P$.

2.2.6. Standard coverings of $\partial \Gamma$ by combinatorial disks. Let $G$ be a group with locally-finite negatively curved Cayley graph $\Gamma$. Fix a base vertex $\mathcal{O}$ for $\Gamma$. 
For each positive integer $n$, define

$$
\mathcal{D}(n)=\{D(R, n) \mid R \text { is a ray in } \Gamma \text { with } R(0)=\mathcal{O}\} .
$$

\subsection{Statement of the main theorem.}

Main Theorem, Theorem 2.3.1. A group $G$ can act properly discontinuously, isometrically, and cocompactly on hyperbolic 3-space $\mathbb{H}^{3}$ if and only if $G$ has a locally finite Cayley graph $\Gamma$ satisfying

1. $\Gamma$ is negatively curved;

2. $\partial \Gamma=\mathbf{S}^{2}$; and

3. the sequence $\mathcal{D}(1), \mathcal{D}(2), \mathcal{D}(3), \ldots$ is conformal.

If these conditions are satisfied with respect to one locally-finite Cayley graph and base vertex $\mathcal{O}$, they are satisfied with respect to all.

Remarks 2.3.2. Theorem 2.3.1 reduces a difficult 3-dimensional problem to a difficult 2-dimensional problem, namely the estimation of combinatorial moduli. The 2-dimensional problem has a combinatorial flavor, as we shall see at the end of Section 3, where we shall prove that the sequence $\mathcal{D}(1), \mathcal{D}(2), \ldots$ is recursively defined by a subdivision rule. The paper [9] is devoted to the study of the estimation of combinatorial moduli. That subject is complex and beautiful.

Tukia [30] and Cannon and Cooper [8] have given an earlier 3-dimensional characterization of the groups of Theorem 2.3.1.

Theorem [8]. A finitely generated group $G$ can act properly discontinuously, isometrically, and cocompactly on hyperbolic 3-dimensional space $\mathbb{H}^{3}$ if and only if a locally-finite Cayley graph $\Gamma$ for $G$ is quasi-isometric with $\mathbb{H}^{3}$.

The theorem follows from Tukia's work, though it is not explicitly stated. The Cannon-Cooper paper has a generalization to finite volume groups which suggests a generalization of Theorem 2.3.1 to finite volume groups.

The major ingredients in our work have two sources: the combinatorial Riemann mapping theorem from [7] and fundamental properties of negatively curved groups and spaces from [27]. Since the material from [27] is not generally available, we include the necessary proofs here, which fill most of Sections 3 and 4.

\section{Negatively Curved SpaCes AND GRoups}

Most of this section appears in Swenson's thesis [27]. We assume that $X$ is a space with a proper path metric that is negatively curved (see Section 1 ). We assume that $\delta$ is a thinness constant for $X$ so that $n$-gons in $X$ are $(n-2) \delta$-thin.

Such spaces were defined in generality by Gromov [15] and have been studied extensively. See, for example, [1], [2], [4], [5], [6], [10], [15], [27] and others.

The geometry of negatively curved spaces is startlingly different from the Euclidean geometry with which most of us are familiar.

First of all, the spaces behave very much like trees. If a result seems unusual, a glance at a tree will usually illustrate the unusual behavior in a concrete setting. Probably the best technique for viewing the negatively curved space as a tree is embedded in a very insightful result of Gromov termed in [10] "lemma of approximation".

Theorem 3.1 [10, Chapter 6, Theorem 1]. Let $X$ be a negatively curved space with thinness constant $\delta$; let $X_{0}=\left\{x_{0}, x_{1}, \ldots, x_{n}\right\}$ be a collection of $n+1$ points of 
$X \cup \partial X$ and let $Y$ denote a union of segments $\left[x_{0}, x_{i}\right]$. Suppose further that $2 n \leq$ $2^{k}+1$. Then there exist a simplicial tree, denoted $\operatorname{Tr}(Y)$, and a continuous function $f: Y \rightarrow \operatorname{Tr}(Y)$ with the following two properties:

1. For each $i$, the restriction of $f$ to $\left[x_{0}, x_{i}\right]$ is an isometry onto its image. In particular, for each point $x \in Y$, the distances $d\left(f(x), f\left(x_{0}\right)\right)$ and $d\left(x, x_{0}\right)$ are equal.

2. For every $x, y \in Y$, one has $d(x, y)-2 k \delta \leq d(f(x), f(y)) \leq d(x, y)$.

In other words, the distance relationships among any finite number of points of $X$ are very well approximated by the distance relationships in some tree $\operatorname{Tr}(Y)$. We shall not make explicit use of this lemma; but, if the reader will attempt to construct the appropriate trees associated with each of our lemmas, it will soon become apparent why the lemma is true or what direction should be pursued in proving the lemma.

Second, negatively curved spaces are not assumed to have any particularly nice local behavior, so that one has to get used to ignoring most local information. Roughly speaking, any behavior at roughly the scale of $\delta$ or one of its small multiples can be ignored without peril.

In summary, any serious theorem-proving must rely on an entire new arsenal of fundamental facts which the uninitiated reader may find unpalatable since there are so many of them. In an attempt to aid the digestion, we collect the facts without interruption for proofs, then give the proofs consecutively at the end of the section. The facts should be studied and contemplated as a whole, independent of the proofs, since they come in related families. After one has a feeling for the nature of these facts, one can either construct proofs or read those given.

Notation. Let $x_{1}, \ldots, x_{n} \in X$. Then $\left[x_{1}, \ldots, x_{n}\right]$ denotes a broken geodesic path with vertices $x_{1}, \ldots, x_{n}$. That is, $\left[x_{1}, \ldots, x_{n}\right]$ is the union of segments $\left[x_{1}, x_{2}\right]$, $\ldots,\left[x_{n-1}, x_{n}\right]$. The closed polygon with the same vertices, $\mathcal{P}\left[x_{1}, \ldots, x_{n}\right]$, differs from $\left[x_{1}, \ldots, x_{n}\right]$ only in the inclusion of a segment joining $x_{1}$ and $x_{n}$.

Positive functions of $\delta$. Our assertions involve positive constants which usually depend only on the thinness constant $\delta$. Exact values for these constants either are given explicitly in the proofs or can be deduced from the arguments given there. Statements are more conceptual and proofs are easier if we do not worry about obtaining the best possible constants.

Fundamental arguments. Let $\mathcal{P}\left[x_{1}, \ldots, x_{n}\right]$ denote an $(n-2) \delta$-thin $n$-gon. The following arguments are used again and again:

Distance-from-projection. If $d\left(x_{n}, x_{1}\right)=d\left(x_{n},\left[x_{1}, x_{2}\right]\right)$, then $x_{1}$ lies within $2(n-2) \delta$ of the broken geodesic, $\left[x_{2}, \ldots, x_{n}\right]$.

Proof. If $d\left(x_{n}, x_{1}\right) \leq(n-2) \delta$, we are done. Otherwise, let $x \in\left[x_{n}, x_{1}\right]$ be at distance $(n-2) \delta$ from $x_{1}$. Points $y \neq x$ near to $x$ in $\left[x_{n}, x_{1}\right]$ are at distance $\leq(n-2) \delta$ of $\left[x_{2}, \ldots, x_{n}\right]$ since $\mathcal{P}\left[x_{1}, \ldots, x_{n}\right]$ is $(n-2) \delta$-thin and $y$ is not within $(n-2) \delta$ of $\left[x_{1}, x_{2}\right]$. By continuity, $x$ is within $(n-2) \delta$ of $\left[x_{2}, \ldots, x_{n}\right]$. Hence

$$
d\left(x_{1},\left[x_{2}, \ldots, x_{n}\right]\right) \leq d\left(x_{1}, x\right)+d\left(x,\left[x_{2}, \ldots, x_{n}\right]\right) \leq 2(n-2) \delta .
$$

Connectedness. If $[a, b] \subset\left[x_{n}, x_{1}\right]$ and $a$ and $b$ are within $(n-2) \delta$ of different sides of $\left[x_{1}, \ldots, x_{n}\right]$, then some point $x \in[a, b]$ is within $(n-2) \delta$ of two sides of $\left[x_{1}, \ldots, x_{n}\right]$. 
Proof. Let $K_{i}$ denote the closed subset of $[a, b]$ consisting of those points within $(n-2) \delta$ of $\left[x_{i}, x_{i+1}\right], i=1, \ldots, n-1$. These sets cover the connected set $[a, b]$; at least two are nonempty; hence at least two intersect.

Distance-to-projection. Suppose $\mathcal{P}\left[x_{1}, \ldots, x_{n}\right]$ is a closed polygon with $x_{1}$ a projection of $x_{n}$ on $\left[x_{1}, x_{2}\right]$. If $x \in\left[x_{1}, x_{2}\right]$ is within $N$ of $\left[x_{n}, x_{1}\right]$, then $d\left(x, x_{1}\right) \leq$ $2 N$. If in addition $x_{2}$ is a projection of $x_{3}$ on $\left[x_{1}, x_{2}\right]$, and if $d\left(x,\left[x_{2}, x_{3}\right]\right) \leq N$, then $d\left(x_{1}, x_{2}\right) \leq 4 N$.

Proof. Clear.

\section{Definitions.}

3.2. A interval $R$ in $X$ is an isometric embedding of a connected subset of the real line $\mathbf{R}$ into $X$. We use the symbol $R$ both for the function and for its image. We are particularly interested in rays, which have $[0, \infty)$ as domain of definition; segments $[x, y]$, which have a finite closed interval as domain with $x$ and $y$ as image endpoints; and lines, which have the entire real line $\mathbf{R}$ as domain. With segments there is some ambiguity in the notation since geodesics joining two points need not be unique; we therefore adopt the convention that, if $x$ and $y$ are already on an interval, then $[x, y]$ is the obvious subpath of that interval; otherwise the choice of the path $[x, y]$ will be immaterial.

3.3. Since closed metric balls in $X$ are compact (the metric is assumed proper), if $C$ is any nonempty closed subset of $X$ and $x$ is any point of $X$, then there is at least one point of $C$ that is as close to $x$ as any other. We call such a point a nearest point projection of $x$ in $C$. If we want to be more formal, or if we want to consider the entire set of nearest points, we may define $\pi(x, C)$ to be the compact, nonempty set of nearest point projections of $x$ in $C$. Half-spaces $H(R, n)$, which we defined in Section 1, may be defined in terms of nearest point projection:

$$
\begin{aligned}
H(R, n) & =\{x \in X \mid d(x, R([n, \infty)) \leq d(x, R([0, n]))\} \\
& =\{x \in X \mid \pi(x, R) \cap R([n, \infty)) \neq \emptyset\} .
\end{aligned}
$$

It is convenient to have the notation $H^{c}(R, n)$ for the complement of $H(R, n)$ in $X$.

\section{Facts and intuitions.}

\section{Projections, segments, and rays.}

3.4. (Set $K=4 \delta:$ ) Nearest point projection is almost unique. For geodesics $R$ and points $x \in X$, the sets $\pi(x, R)$ have diameter less than $K$.

3.5. $\left(\forall K_{1}>0\right.$ set $\left.K_{2}=K_{1}+2 \delta:\right)$ Two segments whose endpoints are less than $K_{1}$ apart (in pairs) are everywhere less than $K_{2}$ apart.

3.6. $\left(\exists K_{1}=8 \delta, \exists K_{2}=24 \delta:\right)$ For intervals $R$ and points $x$ and $y$ having nearest point projections $x^{\prime}$ and $y^{\prime}$ into $R$ with $d\left(x^{\prime}, y^{\prime}\right) \geq K_{1}$, geodesics from $x$ to $y$ roughly follow, in turn, the three segments $\left[x, x^{\prime}\right],\left[x^{\prime}, y^{\prime}\right]$, and $\left[y^{\prime}, y\right]$. In particular the distance from $x$ to $y$ is within $K_{2}$ of the sum of the three segment lengths. 
3.7. $\left(\forall K_{1}>4 \delta \exists K_{2}=K_{2}\left(K_{1}, \delta\right)\right.$ :) Two intervals, which anywhere approach one another closely, viewed together form roughly the capital letter $I$ That is, if two intervals approach one another at some point within $K_{1}$, then the two intervals converge on one another linearly from the four ends (forming the flange ends of the letter I) until they are within $K_{1}$, and then they have subarcs which are uniformly within $K_{2}$ (forming the central arm of the letter I).

3.8. (Set $K=2 \delta:)$ Geodesic rays which are equivalent are not only asymptotically a finite distance apart but asymptotically within $K$ of one another, not as functions but as sets.

3.9. (Set $K=6 \delta:)$ Equivalent rays $R$ and $S$ have strongly correlated parametrizations. That is, there are positive numbers $r$ and $s$ such that, for all positive numbers $t, d(R(r+t), S(s+t)) \leq K$.

3.10. Given any sequence $g_{1}, g_{2}, \ldots$ of intervals all of which hit some compact set $K$, there is a subsequence which converges uniformly on compact subintervals to a interval $R$ with $\ell(R)=\lim \sup \ell\left(g_{i}\right)$. Moreover, if limsup $d\left(K\right.$, endpoint-set $\left.\left(g_{i}\right)\right)=$ $\infty$ then $R$ is a line, where of course $d\left(K\right.$, endpoint-set $\left.\left(g_{i}\right)\right)=\infty$ when $g_{i}$ has no endpoints.

3.11. ( $\forall K>0$ sufficiently large:) Inequivalent rays, $R$ and $S$ may have finite subintervals on which they are within $K$ of one another, but thereafter they diverge metrically essentially as rapidly as their isometric parametrizations allow.

3.12. Given a ray $R$ and a base point $\mathcal{O}$ in $X$, there is a ray $S$ equivalent to $R$ which has initial point $\mathcal{O}$.

3.13. Given two points at infinity, there is a line $L$ joining those two points. That is, $L \mid[0, \infty)$ is a ray representing one of those two points; $L \mid(-\infty, 0]$ represents the other.

\section{Half-spaces.}

3.14. (Set $\left.K_{1}=4 \delta, K_{2}=2 \delta:\right)$ A half-space has a single entrance-exit. That is, if $H(R, n)$ is a half-space, $x$ and $y$ are points of $X$ having nearest-point projections $x^{\prime}$ and $y^{\prime}$ on $R$ with $d\left(x^{\prime}, R(n)\right) \geq K_{1}$ and $d\left(y^{\prime}, R(n)\right) \geq K_{1}$, $x^{\prime}$ and $y^{\prime}$ on opposite sides of $R(n)$ in $R$, then the segment $[x, y]$ intersects the metric ball $B\left(R(n), K_{2}\right)$.

3.15. (Set $K=4 \delta:)$ Deep geodesic penetration $(m \geq 2 K)$ into a half-space $H(R, n+m)$ from outside $H(R, n)$ must proceed along a long narrow entrance corridor defined as a uniform $K$-neighborhood of the segment $R([n+K, n+m-K])$.

3.16. $(\exists K=K(\delta)>0$ :) If $H(R, r)$ and $H(R, r+t)$ are concentric half-spaces, then the distance from $H(R, r+t)$ to the complement $H^{c}(R, r)$ is within $K$ of $t$.

3.17. $(\exists K=K(\delta)>0:)$ Geodesics whose endpoints lie outside a half-space $H(R, n)$ can only penetrate into $H(R, n+m)$ if $m<K$.

Definition. A set $S$ is almost-convex $(K)$ if any segment joining points of $S$ is contained in the $K$ neighborhood of $S$.

3.18. Half-spaces and their complements are uniformly almost-convex $(2 \delta)$.

3.19. $(\exists K=K(\delta)>0$ :) Half-spaces on inequivalent rays can be chosen disjoint. What is required in order that $H(R, r)$ and $H(S, s)$ be disjoint is that $R(r)$ be further than $K$ both from $S$ and from a segment $T$ joining $R(0)$ to $S(0)$, and, similarly, that $S(s)$ be sufficiently far from $R$ and $T$. 
3.20. $\left(\exists K_{1}=6 \delta, \exists K_{2}=K_{2}(\delta)>0\right.$ :) Half-spaces on equivalent rays nest uniformly. That is, if $R$ and $S$ are equivalent rays, and if, for all large $n, R(r+n)$ and $S(s+n)$ are at distance $\leq K_{1}$, then, for all $k \geq K_{2}, H(R, r+n+k) \subset H(S, s+n)$.

3.21. (Set $K=4 \delta:)$ Half-spaces $H(R, r)$ are locally defined. That is, the half-space $H(R, r)$ is determined by a $K$-neighborhood of $R(r)$ in $R$.

3.22. (Set $K=5 \delta:)$ Suppose $R(0)=S(0)$ and suppose $H(R, r) \cap H(S, s) \neq \emptyset$. Then either $R([r, \infty))$ has a point within $K$ of $S$ or $S([s, \infty))$ has a point within $K$ of $R$.

3.23. (Set $K=16 \delta$ :) Suppose $R$ and $S$ are rays with $R(0)=S(0)=\mathcal{O}$. If $S(\infty) \notin D(R, n)$, then

$$
\begin{aligned}
H(R, n+K) & \cap H(S, n+K)=\emptyset . \\
\text { Consequently, } D(R, n+K) & \cap D(S, n+K)=\emptyset .
\end{aligned}
$$

Disks and shingles at infinity. We conjecture that, if $\partial X$ is a 2 -sphere, then the combinatorial disks $D(R, n)$ at infinity are in fact topological open disks. We do not know how to prove our conjecture. On the other hand, the combinatorial Riemann mapping theorem [7] which we wish to apply uses coverings of $\mathbf{S}^{2}$ by compact connected sets, termed shingles. We thus cannot directly apply the coverings $\mathcal{D}(n)$, whose elements are, by construction, open rather than closed and may, conceivably, be disconnected. Our next facts deal with that problem by passing from covers by combinatorial disks to covers by shingles.

3.24. ( $\exists K=K(\delta)>0$ :) Suppose $R$ and $S$ are rays and $S(\infty) \notin D(R, n)$. Then asymptotically $S$ lies within $K$ of $H^{c}(R, n)$.

3.25. (Set $K=12 \delta:$ ) Suppose that $R$ and $S$ are rays with $R(0)=\mathcal{O}=S(0)$. If $d(R(t), S(t))>K$, then $H(R, t) \cap H(S, t)=\emptyset$. In particular, $D(R, t) \cap D(S, t)=\emptyset$.

3.26. $(\exists K=K(\delta)>0$ :) If $m>K$, then the closure of $D(R, n+m)$ lies in $D(R, n)$. Hence we can replace the open set $D(R, n+m)$ by its closure without expanding the size too much.

3.27. Suppose $\partial \Gamma$ is locally connected for $\Gamma$, the locally finite Cayley graph of a negatively curved group $G$. Then there exists a number $m>4 \delta$, such that, for all segments $R:[-4 \delta, m] \rightarrow \Gamma$, there exists a component $C$ of $D(R, 0)$ such that for all segments $R^{\prime}:[-4 \delta, 4 \delta+m] \rightarrow \Gamma$ extending $R, D\left(R^{\prime}, m\right) \subset C$.

3.28. In the setting of (3.27) disks may be expanded to shingles in a canonical way without increasing size too much. Simply replace the disk $D(R, n)$ with the shingle $S(R, n, n-m)$ which is the closure of the component of $D(R, n-m)$ which contains $D(R, n)$. By (3.27), the procedure makes sense. By (3.26), it increases the size a controlled amount.

Finiteness and recursive properties of half-spaces, combinatorial disks, and shinglings. We now restrict our attention to the case where $X=\Gamma$, where $\Gamma$ is a locally-finite Cayley graph for a negatively curved group $G$, and $\partial \Gamma$ is locally connected. We also restrict ourselves to rays $R:[0, \infty) \rightarrow \Gamma$ with initial point $R(0)$ at some fixed vertex $\mathcal{O}$, and to parameters $n$ and $m$ that are positive integers with $m<n$. We then have the natural collections,

$$
\mathcal{H}(n)=\{H(R, n)\}, \text { the combinatorial half-spaces; }
$$




$$
\begin{gathered}
\mathcal{D}(n)=\{D(R, n)\}, \text { the combinatorial disks; and } \\
\mathcal{S}(n, m)=\{S(R, n, n-m)\}, \text { the combinatorial shingles. }
\end{gathered}
$$

Recall that $S(R, n, n-m)$ is the closure of the component of $D(R, n-m)$ containing $R(\infty)$; for $m$ sufficiently large, $S(R, n, n-m)$ contains $D(R, n)$ by $(3.27)$. See also (3.26).

With these special assumptions and restrictions, the three collections are very well-behaved, as the following facts demonstrate.

3.29. If, for the purpose of this proposition only, we let the base point $\mathcal{O}$ vary, then, as the group $G$ acts on the graph $\Gamma$, it also acts on the sets of all combinatorial half-spaces, all combinatorial disks, and all shingles (of fixed parameter $m$ ). In all three cases the action has only finitely many orbits.

3.30. With fixed base vertex $\mathcal{O}$, the sequences $\mathcal{H}(n), \mathcal{D}(n)$, and $\mathcal{S}(n, n-m)$ can be defined by a finite recursion which may be interpreted as a subdivision rule. Since our main theorem is stated in terms of the sequence $\mathcal{D}(n), n=1,2, \ldots$, it follows that the constant curvature of a group $G$ is dependent on the properties of this subdivision rule on the 2 -sphere.

\section{Proofs.}

Proof of 3.4. Construct the following diagram.

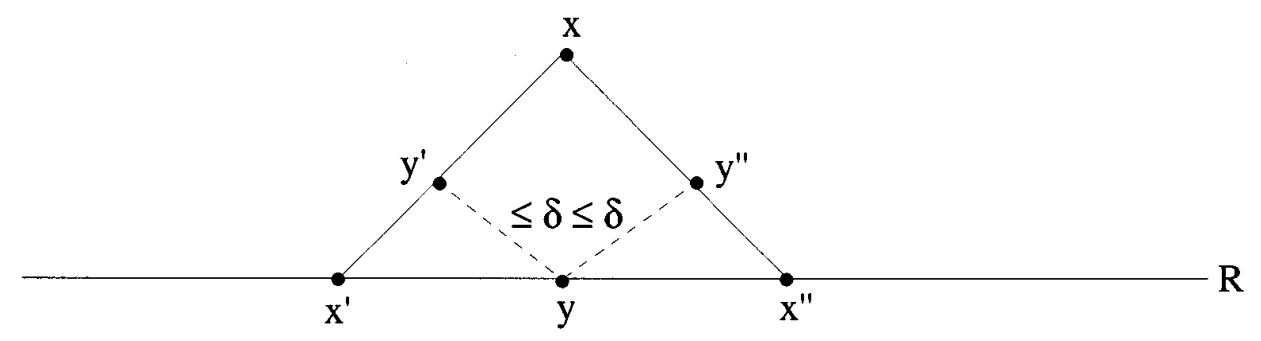

FiguRE 3.4

Let $x^{\prime}$ and $x^{\prime \prime}$ be nearest point projections from $x$ to $R$. By the standard argument of connectedness some point $y$ of $\left[x^{\prime}, x^{\prime \prime}\right]$ lies within $\delta$ of $y^{\prime} \in\left[x, x^{\prime}\right]$ and of $y^{\prime \prime} \in\left[x, x^{\prime \prime}\right]$. By the standard argument of distance-to-projections, $d\left(x^{\prime}, x^{\prime \prime}\right) \leq K=$ $4 \delta$.

Proof of 3.5. Construct the following diagram.

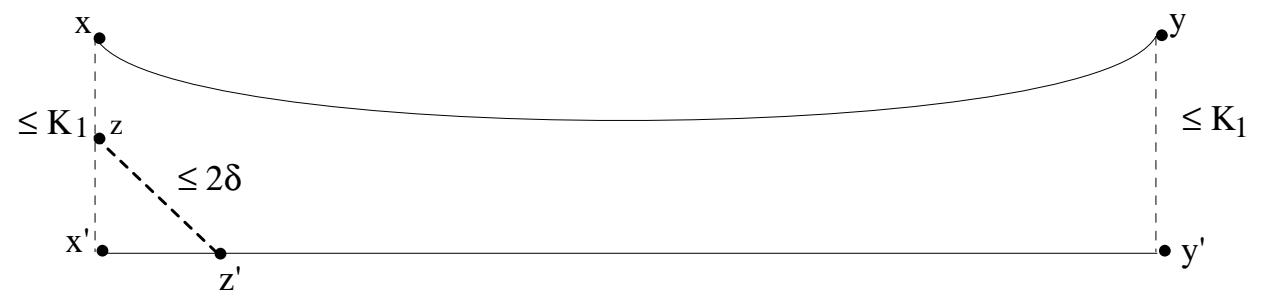

FiguRe 3.5 
Suppose $d\left(x, x^{\prime}\right), d\left(y, y^{\prime}\right) \leq K_{1}$. Let $z^{\prime} \in\left[x^{\prime}, y^{\prime}\right]$. Then $z^{\prime}$ is within $2 \delta$ of $z \in$ $\left[y^{\prime}, y, x, x^{\prime}\right]$, hence within $K_{2}=K_{1}+2 \delta$ of $[x, y]$.

Proof of 3.6. Construct the following diagram.

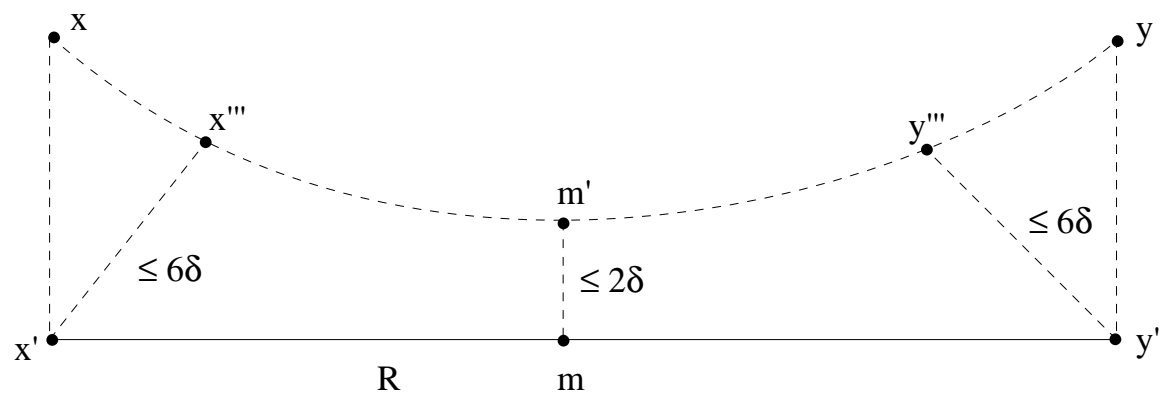

FiguRE 3.6

We set $K_{1}=8 \delta$. If $d\left(x^{\prime}, y^{\prime}\right)>K_{1}$, then the midpoint $m$ of $\left[x^{\prime}, y^{\prime}\right]$ lies at distance $>4 \delta$ from $x^{\prime}$ and $y^{\prime}$. Hence, by distance-to-projection, $m$ lies at distance $>2 \delta$ from $\left[x, x^{\prime}\right]$ and from $\left[y, y^{\prime}\right]$, and consequently $m$ lies $\leq 2 \delta$ from $m^{\prime} \in[x, y]$. By distancefrom-projection, $x^{\prime}$ lies within $4 \delta$ of $x^{\prime \prime}$ in $\left[x, m^{\prime}, m\right]$, hence within $6 \delta$ of $x^{\prime \prime \prime}$ in $\left[x, m^{\prime}\right]$. Similarly, $y^{\prime}$ lies within $6 \delta$ of $y^{\prime \prime \prime}$ in $\left[y, m^{\prime}\right]$. By $(3.5),\left[x, x^{\prime \prime \prime}\right]$ is everywhere within $8 \delta$ of $\left[x, x^{\prime}\right],\left[x^{\prime \prime \prime}, y^{\prime \prime \prime}\right]$ is everywhere within $8 \delta$ of $\left[x^{\prime}, y^{\prime}\right]$, and $\left[y^{\prime \prime \prime}, y\right]$ is everywhere within $8 \delta$ of $\left[y^{\prime}, y\right]$. Also, by the triangle inequality,

$$
\left|d(x, y)-\left[d\left(x, x^{\prime}\right)+d\left(x^{\prime}, y^{\prime}\right)+d\left(y^{\prime}, y\right)\right]\right| \leq 4 \cdot(6 \delta) .
$$

Proof of 3.7. Construct the following diagram.

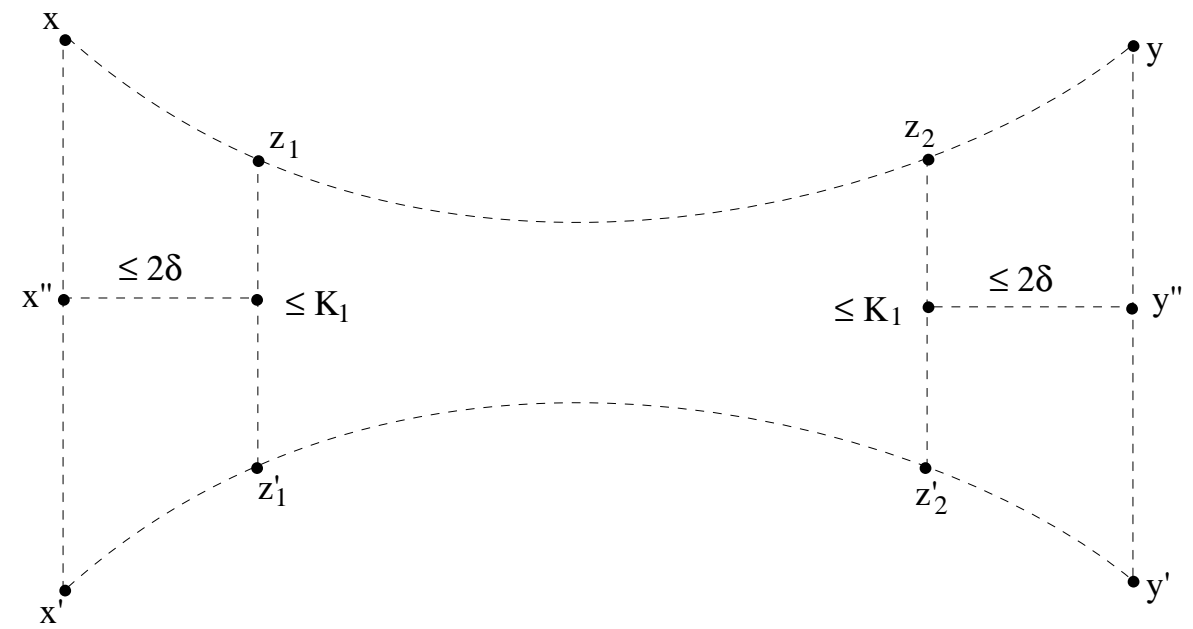

FiguRE 3.7 
Fix $K_{1}>4 \delta$. Let $z_{1}$ be the first point of $[x, y]$ within $K_{1}$ of $\left[x^{\prime}, y^{\prime}\right], z_{2}$ the last, say $d\left(z_{1}, z_{1}^{\prime}\right) \leq K_{1}, d\left(z_{2}, z_{2}^{\prime}\right) \leq K_{1}$. By connectedness there is a point $x^{\prime \prime}$ of $\left[x, x^{\prime}\right]$ within $2 \delta$ of each of two sides of $\left[x, z_{1}, z_{1}^{\prime}, x^{\prime}\right]$. Since $z_{1}$ is the first point of $[x, y]$ within $K_{1}$ of $\left[x^{\prime}, y^{\prime}\right], x^{\prime \prime}$ cannot be within $2 \delta$ of both $\left[x, z_{1}\right]$ and $\left[x^{\prime}, z_{1}^{\prime}\right]$. Hence $x^{\prime \prime}$ is within $2 \delta$ of $\left[z_{1}, z_{1}^{\prime}\right]$. Similarly, $y^{\prime \prime}$ is within $2 \delta$ of $\left[z_{2}, z_{2}^{\prime}\right]$. From (3.5) we conclude that $\left[x, z_{1}\right]$ is near $\left[x, x^{\prime \prime}\right],\left[x^{\prime}, z_{1}^{\prime}\right]$ is near $\left[x^{\prime}, x^{\prime \prime}\right],\left[z_{1}, z_{2}\right]$ is near $\left[z_{1}^{\prime}, z_{2}^{\prime}\right],\left[y, z_{2}\right]$ is near $\left[y, y^{\prime \prime}\right],\left[y^{\prime}, z_{2}^{\prime}\right]$ is near $\left[y^{\prime}, y^{\prime \prime}\right]$.

Proof of 3.8. Construct the following diagram.

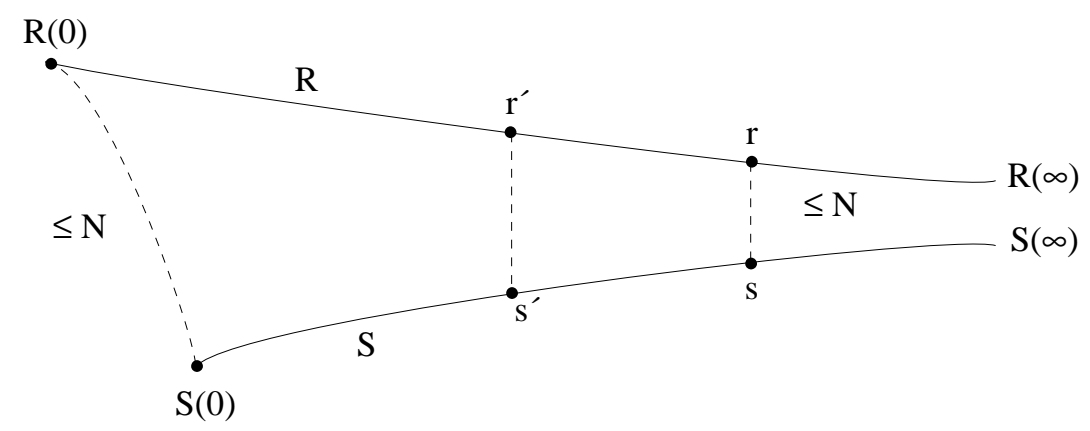

Figure 3.8

Suppose rays $R$ and $S$ are asymptotically within $N$. Suppose $d(R(0), S(0)) \leq N$ as well. Then let $r^{\prime}$ in $R$ be at distance $>N+2 \delta$ from $R(0)$. Pick $r \in\left[r^{\prime}, \infty\right) \subset R$ at distance $>N+2 \delta$ from $r^{\prime}$ such that $d(r, S) \leq N$, say $d(r, s) \leq N$. Then, in the thin quadrilateral $\mathcal{P}[R(0), r, s, S(0)], r^{\prime}$ must be within $2 \delta$ of $s^{\prime} \in[r, s, S(0), R(0)]$. But $r^{\prime}$ cannot be within $2 \delta$ of $[R(0), S(0)] \cup[r, s]$. Hence $s^{\prime} \in S$. We conclude that $R$ is asymptotically within $K=2 \delta$ of $S$.

Proof of 3.9. Construct the following diagram.

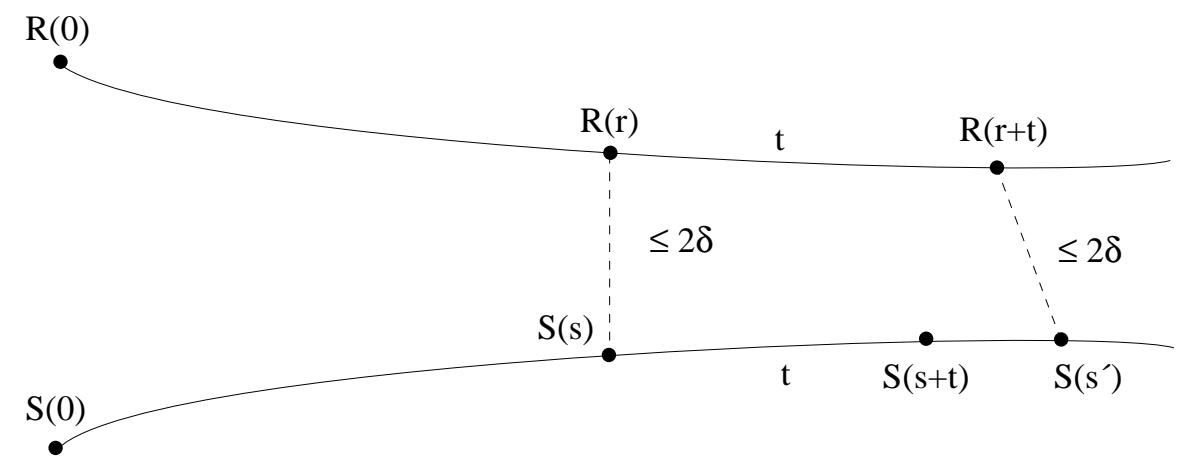

FiguRE 3.9

Let $R$ and $S$ be equivalent rays. By (3.8) there exist points $r, s \in[0, \infty)$ with $d(R(r), S(s)) \leq 2 \delta$ such that for all $r^{\prime}>r$ and $s^{\prime}>s$ we have $d\left(R\left(r^{\prime}\right), S\right) \leq 2 \delta$ and $d\left(R, S\left(s^{\prime}\right)\right) \leq 2 \delta$. For all large values of $t, R(r+t)$ is within $2 \delta$ of some $S\left(s^{\prime}\right)$. 
Comparing [S(s), $\left.S\left(s^{\prime}\right)\right]$ with $[R(r), R(r+t)]$, we find that $d\left(S(s), S\left(s^{\prime}\right)\right)$ is within $4 \delta$ of $t$. Therefore $d\left(S(s+t), S\left(s^{\prime}\right)\right) \leq 4 \delta$ and $d(R(r+t), S(s+t)) \leq 6 \delta$.

Proof of 3.10. We first prove that if $g_{i}=\left[x_{i}, y_{i}\right]$ where $x_{i} \rightarrow x$ and $y_{i} \rightarrow y$, then some subsequence of the sequence of segments $g_{i}$ converges to a segment $[x, y]$ from $x$ to $y$. Parametrize the segments using constant speed such that $g_{i}(0)=x_{i}$ and $g_{i}(1)=y_{i}$. Let $r_{1}, r_{2}, \ldots$ denote an enumeration of the rational numbers in $[0,1]$. Let $G$ denote the sequence of segments. Let $G_{1}$ be a subsequence which omits $g_{1}$ such that the elements of $G_{1}$ converge on $r_{1}$. Let $G_{2}$ denote a subsequence of $G_{1}$ which omits $g_{2}$ such that the elements of $G_{2}$ converge on $r_{2}$. Define subsequences $G_{i}$ for $i>2$ similarly. Let $h_{i}$ denote the first element of the subsequence $G_{i}$, and let $H$ be the sequence of $h_{i}$. Then clearly the sequence $H$ converges on $r_{1}, r_{2}, \ldots$ Since each element of $H$ is a segment, convergence is uniform and $H$ converges on each number in $[0,1]$. It is now easy to see that the limit is a segment from $x$ to $y$.

We now consider the general case. Taking a subsequence if need be, we may assume that $\lim \ell\left(g_{i}\right)=\lim \sup \ell\left(g_{i}\right)$. For each $i$ let $x_{i} \in g_{i} \cap K$. Taking a subsequence if needed, we may assume that $x_{i} \rightarrow x \in K$. Let $\mathcal{C}=$ intervals $S: x \in S$, with some subsequence of subintervals of $\left(g_{i}\right)$ converging to $\left.S\right\}$. Allowing the degenerate interval $[x]$, we see that $\mathcal{C} \neq \emptyset$. Since a nested union of intervals is a interval, by Zorn's Lemma, there is a maximal element $R$ of $\mathcal{C}$. If $\lim \ell\left(g_{i}\right)-\ell(R)>0$, then by taking a subsequence there is a convergent sequence of $y_{i} \in g_{i}$ with $y_{i} \rightarrow y \notin R$. Using the result in the first paragraph, we can extend $R$ to include $y$, contradicting the maximality of $R$, so that $\lim \ell\left(g_{i}\right)=\ell(R)$. Similarly if $\lim \sup d\left(K\right.$, endpoint-set $\left.\left(g_{i}\right)\right)=\infty$, then $R$ cannot have an endpoint, and so is a line.

Proof of 3.11. Let $K=\max \{4 \delta, d(R(0), S(0))\}$. If the set of positive numbers $t$ for which $d(R(t), S) \leq K$ were unbounded, then by (3.5) every point of $R$ would lie within some bounded distance of $S$, and so $S$ and $R$ would be equivalent. Since they are not, there is a last point $r \in R$ with $d(S, r) \leq K$. Similarly there is a last $s \in S$ with $d(s, R) \leq K$. Now apply (3.7).

Proof of 3.12. Construct the following diagram.

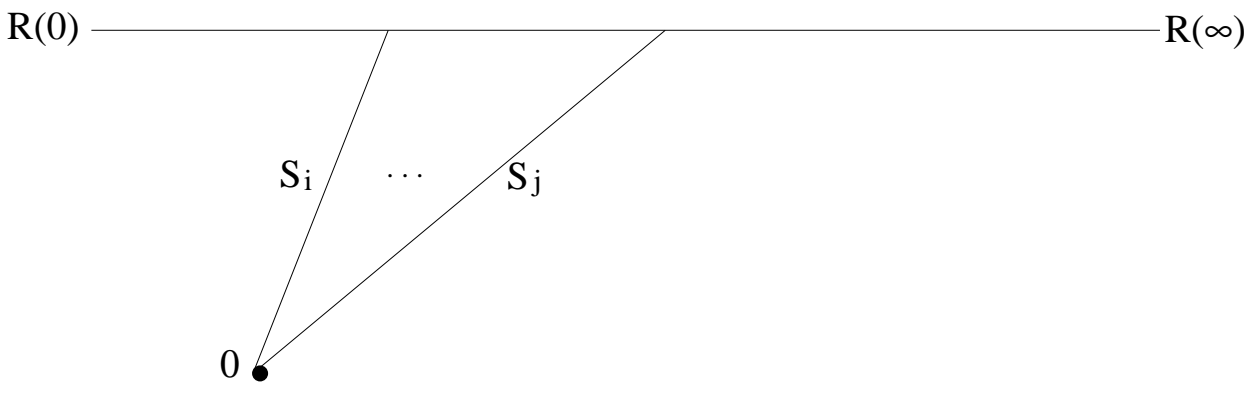

FiguRE 3.12

Let $S_{i}$ denote a segment $[\mathcal{O}, R(i)]$. By (3.10), there is a subsequence of the sequence $S_{1}, S_{2}, \ldots$ which converges to a ray $S$ which will have $\mathcal{O}$ as its endpoint. Applying (3.5) to $R$ and $S_{i}$ shows that every point of $S$ lies within some bounded distance of $R$, and so $S$ and $R$ are equivalent. 
Proof of 3.13. Given two points at infinity, we may assume them represented by rays $R$ and $S$ such that $R(0)=S(0)=\mathcal{O}$, by (3.12). Since $R(\infty) \neq S(\infty)$, by (3.11) all the points of $R$ within $2 \delta$ of $S$ lie in a compact neighborhood $C$ of $\mathcal{O}$. Let $T_{i}=[R(i), S(i)]$. By connectedness, some point $t_{i}$ of $T_{i}$ lies within $\delta$ of both $R$ and $S$, hence in a $\delta$-neighborhood of $C$. By (3.10), some subsequence of the segments $T_{i}$ converges to a line $T$. Since every point of $T_{i}$ is within $\delta$ of $R \cup S$, the same is true of $T$ and we may parametrize $T$ so that the negative end of $T$ is a ray representing $R(\infty)$, while the positive end of $T$ represents $S(\infty)$.

Proof of 3.14. The argument is that of (3.6). Just as $m$ in that proof was within $2 \delta$ of $[x, y]$, so also, in this proof, $R(n)$ is within $2 \delta$ of $[x, y]$.

Proof of 3.15. Let $L$ be a geodesic which penetrates $H(R, n+m)$ from $H^{c}(R, n)$. Use (3.14) to show $B(R(n+K), 2 \delta) \cap L \neq \emptyset$ and $B(R(n+m-K), 2 \delta) \cap L \neq \emptyset$. Apply (3.5) to the subinterval of $L$ from $B(R(n+K), 2 \delta)$ to $B(R(n+m-K), 2 \delta)$.

Proof of 3.16. Choose $K_{1}, K_{2}$ as in (3.6), and set $K=\max \left\{K_{1}, K_{2}\right\}$. If $t \leq K$ there is nothing to prove, so we may assume $t>K$. Let $x \in H^{c}(R, n)$ and $y \in H(R, n+t)$. Applying (3.6) to $[x, y]$ yields $d(x, y) \geq t-K_{2} \geq t-K$, which proves $(3.16)$

Proof of 3.17. This is an easy consequence of (3.15).

Proof of 3.18. Construct the following diagram.

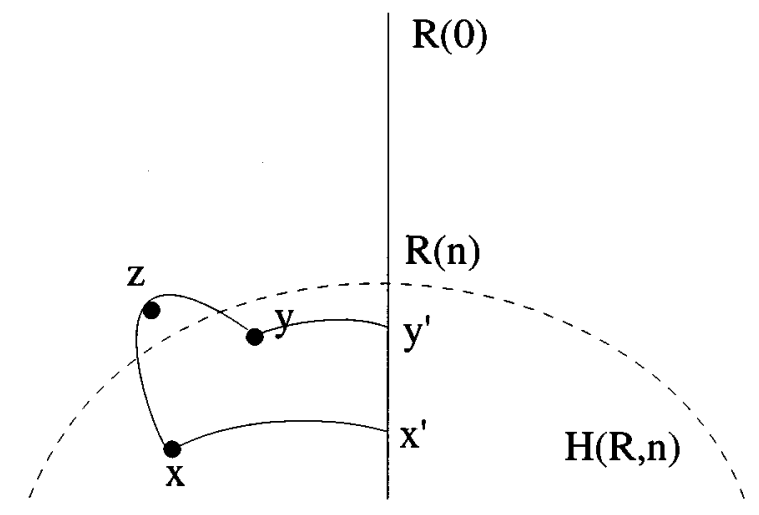

FIGURE 3.18

Let $x$ and $y$ in $H(R, n)$ have projections $x^{\prime}, y^{\prime} \in R([n, \infty))$. Every point $z \in[x, y]$ lies within $2 \delta$ of the union

$$
\left[x, x^{\prime}\right] \cup\left[x^{\prime}, y^{\prime}\right] \cup\left[y^{\prime}, y\right] \subset H(R, n) .
$$

Similarly, $H^{c}(R, n)$ is almost convex. 
Proof of 3.19. Construct the following diagram.

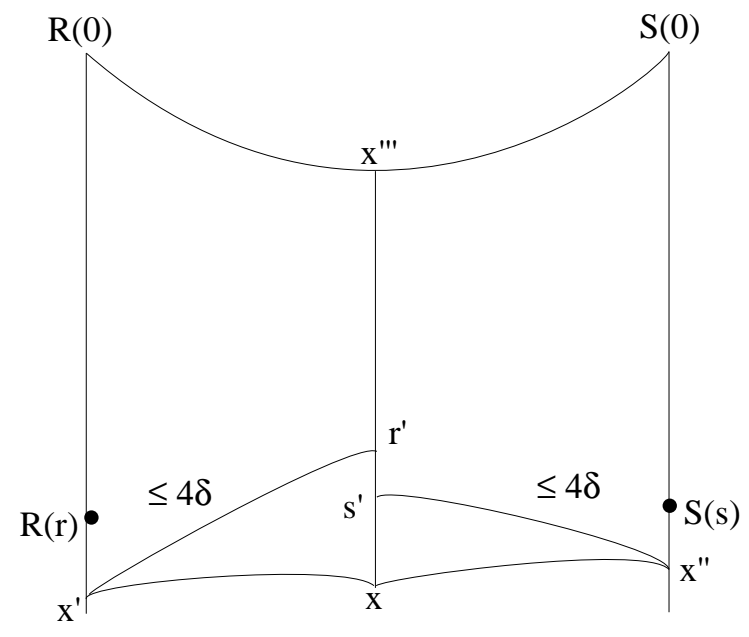

FiguRE 3.19

Let $x \in H(R, r) \cap H(S, s)$. Let $x^{\prime}, x^{\prime \prime}, x^{\prime \prime \prime}$ be nearest point projections into $R, S$, and $T$, respectively. Let $r^{\prime}$ and $s^{\prime}$ be nearest point projections of $x^{\prime}$ and $x^{\prime \prime}$ into $\left[x, x^{\prime \prime \prime}\right]$. We assume $r$ and $s$ so large that $d\left(x^{\prime}, T\right)$ and $d\left(x^{\prime \prime}, T\right)$ are $>4 \delta$. By distance-from-projection applied to $\mathcal{P}\left[x^{\prime}, R(0), x^{\prime \prime \prime}, x\right]$ and $\mathcal{P}\left[x^{\prime \prime}, S(0), x^{\prime \prime \prime}, x\right]$, $d\left(x^{\prime}, r^{\prime}\right)$ and $d\left(x^{\prime \prime}, s^{\prime}\right)$ are $\leq 4 \delta$. We may assume that $r^{\prime}$ lies above $s^{\prime}$ on $\left[x, x^{\prime \prime \prime}\right]$. Then $r^{\prime}$ is within $2 \delta$ of $\left[x^{\prime \prime \prime}, S(0), x^{\prime \prime}, s^{\prime}\right]$. Consequently $x^{\prime}$ is within $4 \delta+2 \delta+4 \delta=10 \delta$ of $\left[x^{\prime \prime \prime}, S(0), x^{\prime \prime}\right]$.

Proof of 3.20. By (3.9) we reparametrize $R$ and $S$ so that, for all $t \geq 0, d(R(t), S(t))$ $\leq K_{1}$. Take $K$ as in (3.16) and let $M=K_{1}+K$. Fix $s>M$. Since $d\left(R(0), H^{c}(S, 0)\right)$ $\leq K_{1}$, by $(3.16) R(0) \in H^{c}(S, s)$. Construct Figure 3.20 .

Let $x \in H^{c}(S, s)$, and let $R(r)$ be a projection of $x$ into $R$. By distance-fromprojection applied to $\mathcal{P}[R(0), x, R(r)], d(R(r),[R(0), x]) \leq 2 \delta$. By (3.18) every point of $[R(0), x]$ is within $2 \delta$ of $H^{c}(S, s)$, and so $d\left(R(r), H^{c}(S, s)\right) \leq 4 \delta$. Using (3.16), $R(r) \notin H\left(S, s^{\prime}\right)$ for $s^{\prime}>s+4 \delta+K$. Since $d(R(t), H(S, t)) \leq K_{1}$, by (3.16) $R(t) \in H\left(S, s^{\prime}\right)$ for all $t \geq s^{\prime}+M$. It follow that $r<s^{\prime}+M$. Thus $x \notin H\left(R, s+K_{2}\right)$ for $K_{2}>M+4 \delta+K$. This implies $H\left(R, s+K_{2}\right) \subset H(S, s)$. The result follows.

Proof of 3.21. We show that if $R$ and $S$ are intervals that agree on $[-4 \delta, 4 \delta]$, then $H(R, 0)=H(S, 0)$. Construct Figure 3.21.

We identify the common image of $[-4 \delta, 4 \delta]$ with the interval $[-4 \delta, 4 \delta]$ of real numbers. We take $x \in H(S, 0)$ and prove $x \in H(R, 0)$. Suppose on the contrary that $x \notin H(R, 0)$. Let $r$ be a projection of $x$ in $R, s$ a projection of $x$ in $S$, $r \in R((-\infty, 0)), s \in S([0, \infty))$.

If $r \in[-4 \delta, 0)$ or if $s \in[0,4 \delta]$, then $[r, s]$ lies in $S$ or in $R$, respectively, so that $[r, s]$ is an edge of a triangle $\mathcal{P}[x, r, s]$. Some point of $[r, s]$ lies by connectedness within $\delta$ of each of $[x, r]$ and $[x, s]$ so that, by distance-to-projection, $d(r, s) \leq 4 \delta$. Hence both $r$ and $s$ lie in $[-4 \delta, 4 \delta]$. Thus $d(x, r)=d(x, s)$, so that $s$ is a projection of $x$ on $R$. Hence $x \in H(R, 0)$. 


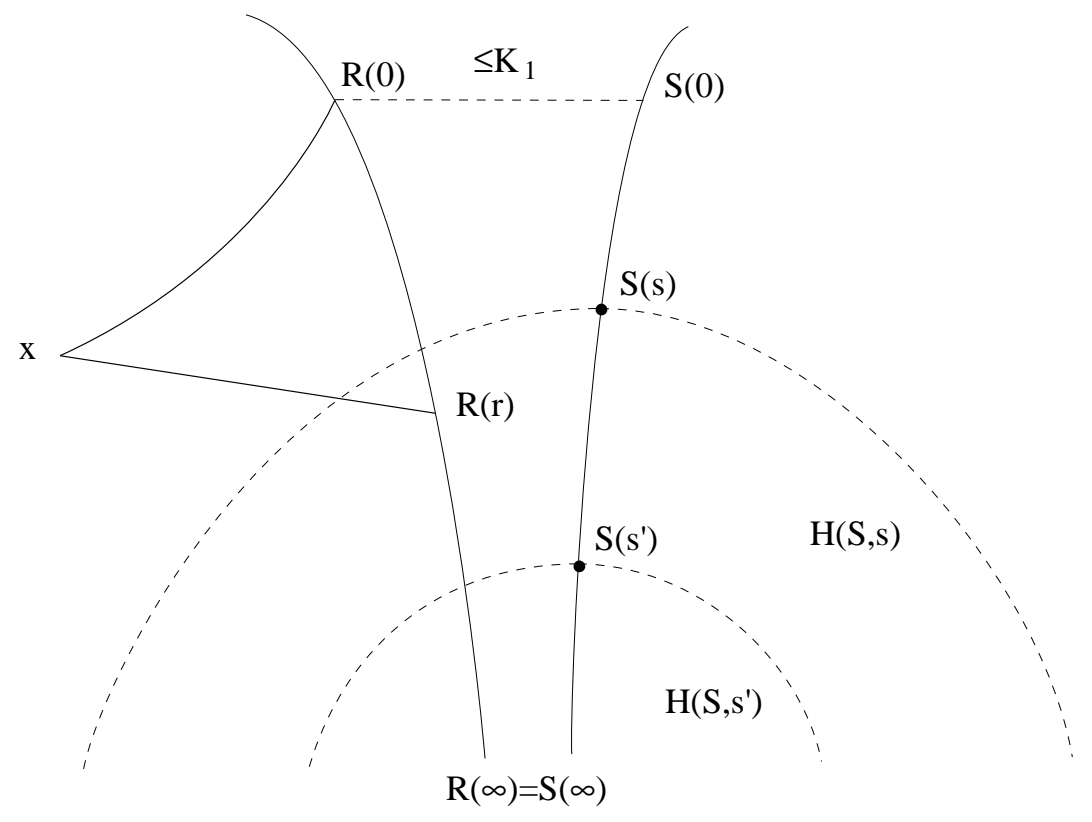

FiguRE 3.20

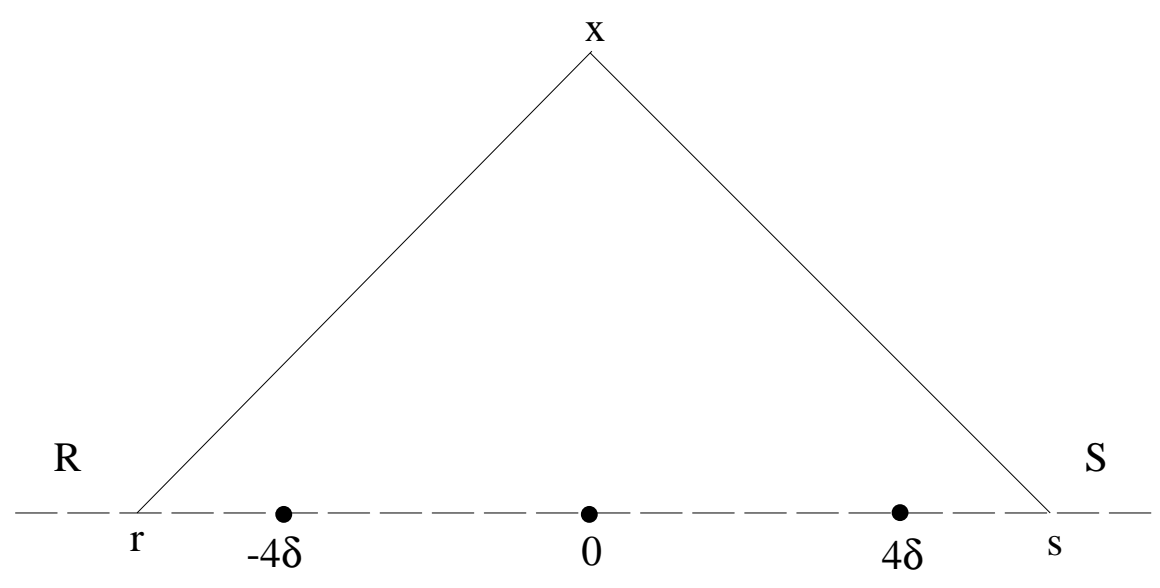

FigURE 3.21

If neither $r$ nor $s$ lies in $[-4 \delta, 4 \delta]$, then consider the quadrilateral $\mathcal{P}[r, 4 \delta, s, x]$. Since $d(0,[4 \delta, s])=4 \delta$, by thin quadrilaterals 0 lies within $2 \delta$ of $[x, r] \cup[x, s]$. With no loss of generality $d(0,[x, s]) \leq 2 \delta$. By distance-to-projection $d(0, s) \leq 4 \delta$, which is a contradiction. 
Proof of 3.22. Construct the following diagram.

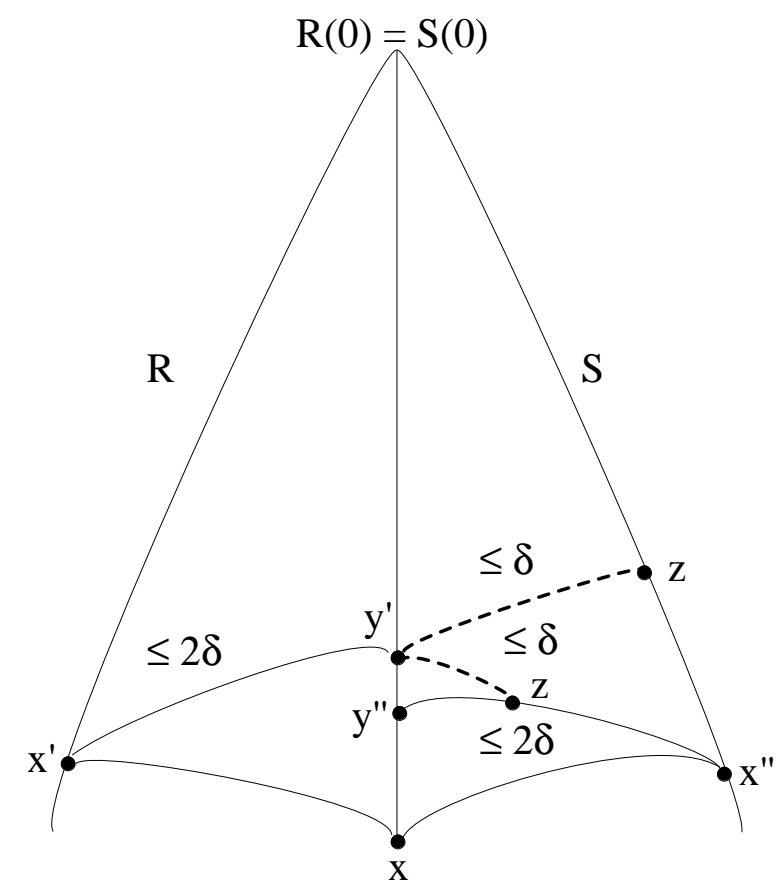

FiguRE 3.22

Let $x \in H(R, r) \cap H(S, s)$ have projections $x^{\prime} \in R([r, \infty))$ and $x^{\prime \prime} \in S([s, \infty))$. Then $x^{\prime}$ is within $2 \delta$ of a point $y^{\prime} \in[R(0), x]$ and $x^{\prime \prime}$ is within $2 \delta$ of a point $y^{\prime \prime} \in[R(0), x]$. We may assume $y^{\prime \prime} \in\left[x, y^{\prime}\right]$. Then $y^{\prime}$ lies within $\delta$ of $z \in\left[x^{\prime \prime}, y^{\prime \prime}\right] \cup$ $\left[S(0), x^{\prime \prime}\right]$. We conclude that $d\left(x^{\prime}, S\right) \leq d\left(x^{\prime}, y^{\prime}\right)+d\left(y^{\prime}, z\right)+d(z, S) \leq 2 \delta+\delta+2 \delta$ $=5 \delta$.

Proof of 3.23. Suppose to the contrary that there exists a point $x \in H(R, n+K) \cap$ $H(S, n+K)$. Construct Figure 3.23.

Points $x^{\prime}$ and $x^{\prime \prime}$ are nearest point projections of $x$ in $R$ and $S$, respectively. The point $y$ is a point of $S$ far beyond $x^{\prime \prime}$ that is near a point $y^{\prime}$ of $H^{c}(R, n)$. Since $S(\infty) \notin D(R, n)$, we may choose $y$ as near $S(\infty)$ as we wish and yet keep $d\left(y, y^{\prime}\right)$ bounded by some uniform constant $L$. Point $y^{\prime \prime}$ is a nearest point projection of $y^{\prime}$ in $R$, necessarily in $R([0, n))$.

We examine the hexagon $\mathcal{P}\left[x^{\prime}, y^{\prime \prime}, y^{\prime}, y, x^{\prime \prime}, x\right]$. By distance-from-projection, $y^{\prime \prime}$ is within $8 \delta$ of a point $z$ of either $\left[y^{\prime}, y\right],\left[y, x^{\prime \prime}\right],\left[x^{\prime \prime}, x\right]$, or $\left[x, x^{\prime}\right]$. We shall show that $z$ cannot be in any of these sets, a contradiction.

If $z \in\left[y^{\prime}, y\right]$, then $d\left(y^{\prime \prime}, y\right) \leq 8 \delta+L$, a contradiction provided that $y$ is sufficiently near $S(\infty)$.

If $z \in\left[y, x^{\prime \prime}\right]$, then $d(S(0), z) \leq n+8 \delta$, a contradiction.

If $z \in\left[x^{\prime \prime}, x\right]$, then $\left[x^{\prime \prime}, x\right] \subset H(S, n+16 \delta)$ is within $n+8 \delta$ of $S(0)$; but $d(S(0), H(S, n+16 \delta)) \geq n+14 \delta$ by the argument for $(3.6)$, a contradiction. The case with $z \in\left[x, x^{\prime}\right]$ is similar but with $R$ instead of $S$. 


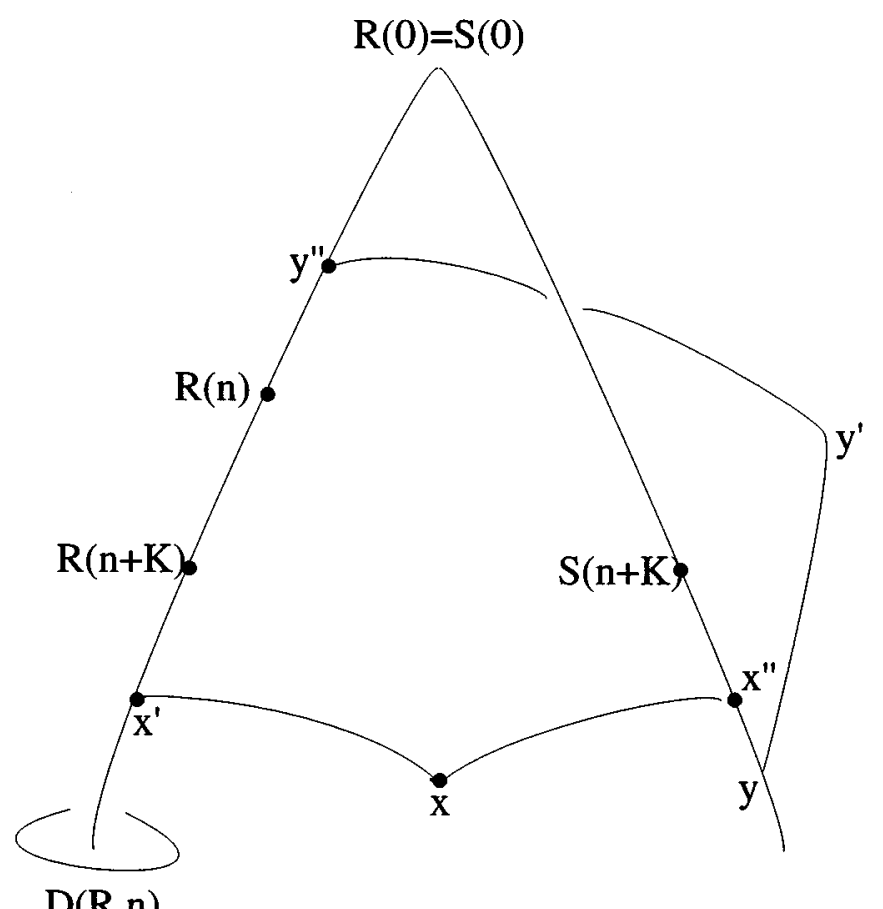

FiguRE 3.23

Proof of 3.24. Construct Figure 3.24.

There exists a positive number $L$ such that

$$
\liminf _{t \rightarrow \infty} d\left(S(t), H^{c}(R, n)\right)<L .
$$

Pick one point $t_{0}$ such that $d\left(S\left(t_{0}\right), H^{c}(R, n)\right) \leq L$ and let $t_{1}$ be very large, such that $d\left(S\left(t_{1}\right), H^{c}(R, n)\right) \leq L$. Choose $u_{0} \in H^{c}(R, n)$ with $d\left(u_{0}, S\left(t_{0}\right)\right) \leq L$, and $u_{1} \in H^{c}(R, n)$ with $d\left(u_{1}, S\left(t_{1}\right)\right) \leq L$. Except for points near $S\left(t_{0}\right)$ and $S\left(t_{1}\right)$, by thin quadrilaterals $S(t)$ must lie within $2 \delta$ of $\left[u_{0}, u_{1}\right]$, which in turn must lie within $2 \delta$ of $H^{c}(R, n)$ by (3.18). We conclude that $S$ lies asymptotically within $4 \delta$ of $H^{c}(R, n)$.

Proof of 3.25. Suppose there exists an $x \in H(R, t) \cap H(S, t)$, and construct Figure 3.25 .

The points $x^{\prime}$ and $x^{\prime \prime}$ are nearest point projections of $x$ into $R$ and $S, x^{\prime} \in$ $R([t, \infty)), x^{\prime \prime} \in S([t, \infty))$. The points $y^{\prime}$ and $y^{\prime \prime}$ are nearest point projections of $x^{\prime}$ and $x^{\prime \prime}$ into $[\mathcal{O}, x], \mathcal{O}=R(0)=S(0)$. We may choose notation so that $y^{\prime} \in\left[\mathcal{O}, y^{\prime \prime}\right]$.

By the argument of $(3.22), x^{\prime}$ is within $5 \delta$ of some $z^{\prime} \in\left[\mathcal{O}, x^{\prime \prime}\right]$.

In the (thin) triangle $\mathcal{P}\left[\mathcal{O}, x^{\prime}, z^{\prime}\right], R(t)$ is within $\delta+5 \delta=6 \delta$ of $\left[\mathcal{O}, z^{\prime}\right]$. Since both $S(t)$ and $R(t)$ are on the same sphere about $\mathcal{O}$, and since $d(R(t), S) \leq 6 \delta$, it follows that $d(R(t), S(t)) \leq 2(6 \delta)=12 \delta$, a contradiction.

Proof of 3.26. Set $K=16 \delta$. Suppose $S$ is a ray with $S(0)=R(0)$ and $S(\infty) \notin$ $D(R, n)$. By (3.23), $D(R, n+K) \cap D(S, n+K)=\emptyset$. In particular, $S(\infty)$ is not an element of the closure of $D(R, n+K)$. That is, the closure lies in $D(R, n)$. 


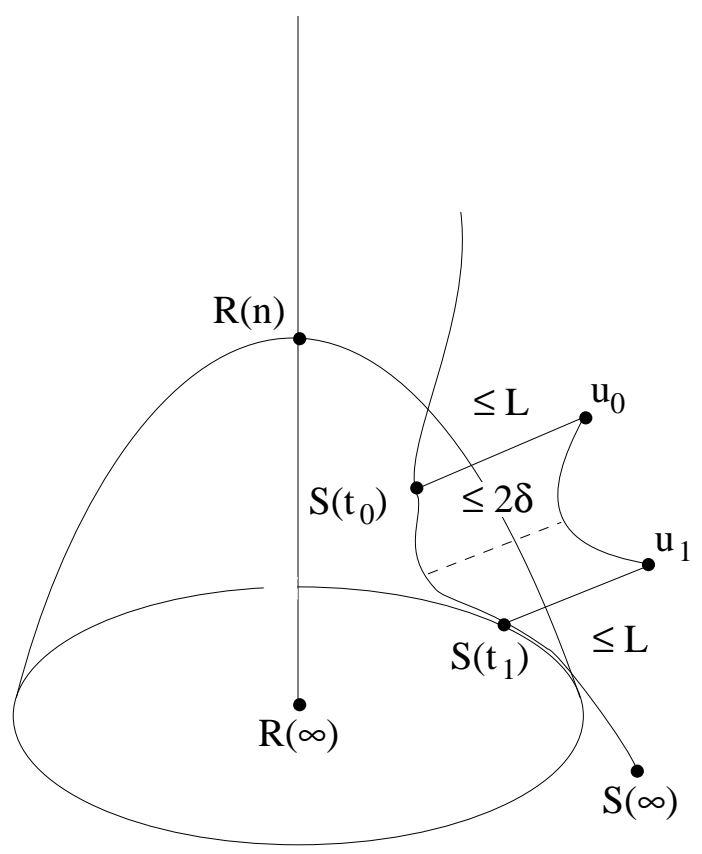

FigURE 3.24

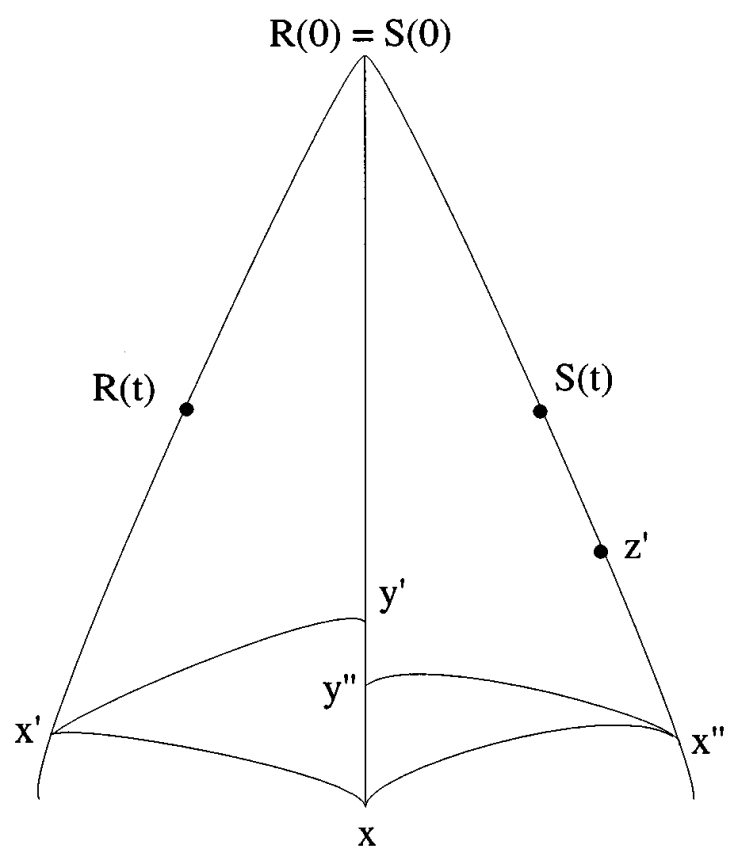

FiguRE 3.25 
Proof of 3.27. Suppose the contrary. Then for each integer $m>4 \delta$ there exists geodesics $R_{m}$ and $\hat{R}_{m}$ with $\left.R_{m}\right|_{[-4 \delta, m]}=\left.\hat{R}_{m}\right|_{[-4 \delta, m]}$ such that $D\left(R_{m}, m\right)$ and $D\left(\hat{R}_{m}, m\right)$ do not lie in the same component of $D\left(R_{m}, 0\right)$ (which is $D\left(\hat{R}_{m}, 0\right)$ by $(3.21))$.

We observe that, translating by $G$, we may assume $R_{m}(0)=R_{i}(0)$ for each $m, i>4 \delta$. Also, by virtue of (3.21), the pair $R_{m}, \hat{R}_{m}$ could be used in place of the pair $R_{i}, \hat{R}_{i}$ for any $4 \delta<i \leq m$.

Using these two observations and passing to a subsequence if necessary, we may assume by (3.10) that there is a ray $R:[-4 \delta, \infty) \rightarrow \Gamma$ such that $\left.R_{m}\right|_{[-4 \delta, m]}=$ $\left.R\right|_{[-4 \delta, m]}$ for each $m$. The component $C$ of $D(R, 0)$ containing $R(\infty)$ is open (by local connectivity); hence for some $N \gg 0, D(R, N) \subset C$ since the disks $D(R, n)$ form a neighborhood basis at $R(\infty)$. For all $m \geq N+4 \delta, D(R, N)=$ $D\left(R_{m}, N\right)=D\left(\hat{R}_{m}, N\right)$ by (3.21). Clearly $D\left(R_{m}, m\right) \subset D\left(R_{m}, N\right) \subset C$ and $D\left(\hat{R}_{m}, m\right) \subset D\left(\hat{R}_{m}, N\right) \subset C$. This contradicts the choice of $R_{m}$ and $\hat{R}_{m}$.

Proof of 3.28. The proof was indicated in the statement. It followed from (3.26) and (3.27).

Proof of 3.29. By (3.21), half-spaces, hence combinatorial disks, are determined by relatively short subsegments of rays, which we may take to have vertices of $\Gamma$ as endpoints. There are only finitely many combinatorial types of such segments. Moreover by (3.27) we see that the same is true of combinatorial shingles. Result (3.29) follows immediately.

Proof of 3.30. We shall construct a multi-valued function from our collections into a finite parameter space involving two parameters. The subdivision rule will be seen to reside essentially in this finite parameter space.

The first parameter is a geodesic word in the Cayley graph $\Gamma$ of fixed length $\mathrm{m}^{\prime}$, where $m^{\prime}$ is chosen as follows. By (3.27), there is an integer $m$ so large that, if $R:[-4 \delta, 4 \delta+m] \rightarrow \Gamma$ is a segment, then $D(R, m)$ lies in a single component of $D(R, 0)$. We may assume that $\delta$ is a positive integer and set $m^{\prime}=8 \delta+m$. Notice that there are only finitely many geodesic words of length $m^{\prime}$.

For our second parameter, we choose the cone-type of a vertex of $\Gamma$. Recall that cone-type was introduced in [5] as follows. The cone $C(x, \mathcal{O})$ at a vertex $x \in \Gamma$ relative to the base vertex $\mathcal{O} \in \Gamma$ is the union of all points $x^{\prime} \in \Gamma$ that can be joined to $\mathcal{O}$ by a segment which passes through $x$. That is, $C(x, \mathcal{O})$ is the shadow of $x$ in $\Gamma$ cast by a light placed at $\mathcal{O}$. Two vertices $x, y \in \Gamma$ are said to have the same cone-type if left multiplication $y x^{-1}: \Gamma \rightarrow \Gamma$ takes $C(x, \mathcal{O})$ isomorphically to $C(y, \mathcal{O})$. By [5] or [6], a negatively-curved group has only finitely many cone-types.

Now let $R:[0, \infty) \rightarrow \Gamma$ denote a ray with $R(0)=\mathcal{O}$. Let $n$ be an integer $>m+4 \delta$. How do we parametrize $H(R, n), D(R, n)$, and $S(R, n, n-m)$ ? For the geodesic-word parameter $W$ we take the group label on the segment

$$
R \mid[n-(m+4 \delta), n+4 \delta] .
$$

For the cone-type parameter $C$ we take the cone-type of the terminal point $R(n+4 \delta)$ of the segment. A given $H(R, n)$ (or $D(R, n)$ or $S(R, n, n-m))$ may arise from infinitely many different rays $R$, but only from finitely many parametrizations $(W, C)$. 
How is subdivision defined? Let $e$ denote an edge beginning at $R(n+4 \delta)$ which lies in some extension $R^{\prime}$ of $R \mid[0, n+4 \delta]$ to a ray. The finitely many labels which will occur on such edges $e$ are completely determined by the cone-type $C$. Let $W^{\prime}$ denote the geodesic word formed from $W$ by deleting the first letter of $W$ and adjoining the label of $e$. Let $C^{\prime}$ denote the cone type of the terminal endpoint of $e$. Then the parametrization corresponding to $H\left(R^{\prime}, n+1\right), D\left(R^{\prime}, n+1\right)$, and $S\left(R^{\prime}, n+1, n+1-m\right)$ is $\left(W^{\prime}, C^{\prime}\right)$. That is, sets with parametrization $(W, C)$ at depth $n$ all lead to sets at depth $n+1$ with parametrizations of the finitely many types $\left(W^{\prime}, C^{\prime}\right)$ associated with $(W, C)$. The proof is complete.

\section{Constant-Curvature groups have COnformal Disk SEQuences}

We prove in this section one half of the main theorem. To that end, we assume that $G$ is a group with a locally finite Cayley graph $\Gamma$ quasi-isometric with hyperbolic 3-space $\mathbb{H}^{3}$. (It follows from Tukia [30], or as a special case of Cannon-Cooper [8], that $G$ acts isometrically, properly discontinuously, and cocompactly on $\mathbb{H}^{3}$, but we shall not make use of that fact. This paper will, in the end, supply another (difficult) proof of that fact.) Consequently, $G$ is negatively curved and has $\partial G=\mathbf{S}^{2}$. We fix a base vertex $\mathcal{O}$ for $\Gamma$ and recall the combinatorial disk cover of $\partial G=\mathbf{S}^{2}$ defined by

$$
\mathcal{D}(n)=\{D(R, n) \mid R(0)=\mathcal{O}\} .
$$

Our goal in this section is to show that the sequence $\mathcal{D}(1), \mathcal{D}(2), \ldots$ of disk covers is conformal in the sense of 2.2 .5 of Section 2 .

4.1. Theorem. If $\Gamma$ is quasi-isometric with $\mathbb{H}^{3}$, then the sequence $\mathcal{D}(1), \mathcal{D}(2), \ldots$ of combinatorial disk covers of $\mathbf{S}^{2}=\partial \Gamma$ is conformal.

The proof will appear in Subsection 4.3, after preparation.

4.2. Almost-round covers by pairs. A pair $\left(D, D^{\prime}\right)$ of subsets, $D \supset D^{\prime}$, of $\mathbf{S}^{2}$ is said to be almost-round $(K)$ if there exist $p \in D^{\prime}$ and $r \geq r^{\prime}>0, r / r^{\prime} \leq K$, such that the $r^{\prime}$-neighborhood $N\left(p, r^{\prime}\right)$ of $p$ in $\mathbf{S}^{2}$ lies in $D^{\prime}$ while $D \subset N(p, r)$. A finite collection of pairs $\left(D_{1}, D_{1}^{\prime}\right), \ldots,\left(D_{k}, D_{k}^{\prime}\right)$ in $\mathbf{S}^{2}$ is said to be a pair-cover of $\mathbf{S}^{2}$, if the larger sets $D_{1}, \ldots, D_{k}$ cover $\mathbf{S}^{2}$ while the smaller sets $D_{1}^{\prime}, \ldots, D_{k}^{\prime}$ are pairwise disjoint. If each of the pairs $\left(D_{i}, D_{i}^{\prime}\right)$ is almost-round $(K)$, then the cover is called almost round. In application, the sets $D_{i}$ and $D_{i}^{\prime}$ will be disks or combinatorial disks.

There is a hyperbolically natural way to obtain almost round sets. Consider $\mathbf{S}^{2}$ as the space at infinity for hyperbolic 3 -space $\mathbb{H}^{3}$. Use the Poincaré disk model for $\mathbb{H}^{3}$. Consider rays $R$ in $\mathbb{H}^{3}$ with initial point at the origin. The combinatorial disks $D(R, n)$ are then, in fact, round spherical disks in $\mathbf{S}^{2}$ with geometric center $R(\infty)$. A pair $(D(R, n), D(R, n+k))$ is almost-round $(K)$ with a $K$ which depends only on the parameter $k$. Any pair $\left(D, D^{\prime}\right)$ such that $D(R, n+k) \subset D^{\prime}$ and $D \subset D(R, n)$ will also be almost round $(K)$. We leave the proofs to the reader.

If one uses a base point other than the origin for the rays in question, then the pairs $(D(R, n), D(R, n+k))$ will still be almost round $\left(K^{\prime}\right)$ for a different $K^{\prime}$ that depends both on the base point and on $k$. However, for $n$ sufficiently large, the parameter $K^{\prime}$ can be taken as near to $K$ as desired. The reason for this stability under change of base point is that almost roundness $K+\epsilon$ is asymptotically invariant under conformal mappings. Again, we leave the proof to the reader. 
4.2.1. Theorem. Almost-round covers by pairs define combinatorial moduli comparable with analytic moduli. Suppose that $K>0$. Then there is a positive number $L$ depending only on $K$ such that, if $\mathcal{R} \subset \mathbf{S}^{2}$ is a ring (annulus) and if $\left(D_{1}, D_{1}^{\prime}\right)$, $\ldots,\left(D_{k}, D_{k}^{\prime}\right)$ is a sufficiently fine pair-cover of $\mathcal{R}$, with the cover almost round $K$, then the combinatorial moduli $M$ and $m$ of $\mathcal{R}$ defined by $D_{1}, \ldots, D_{k}$ and the analytic modulus of $\mathcal{R}$ defined by the standard analytic conformal structure of $\mathbf{S}^{2}$ differ by a factor no bigger than $L$.

4.2.2. Theorem. Negatively curved groups define natural covers at infinity by combinatorial disk-pairs. If $G$ is a negatively curved group and $\Gamma$ a locally finite Cayley graph for $G$, then there is a $k$ sufficiently large that, for all $n$, there is a paircovering of $\partial \Gamma$ by combinatorial disk-pairs of the form $\left(D\left(R_{1}, n\right), D\left(R_{1}, n+k\right)\right), \ldots$, $\left(D\left(R_{j}, n\right), D\left(R_{j}, n+k\right)\right)$. That is, the combinatorial disks $D\left(R_{i}, n+k\right)$ are disjoint while the combinatorial disks $D\left(R_{i}, n\right)$ cover $\partial \Gamma$.

4.2.3. Theorem. The natural covers by disk-pairs defined by a group $G$, with locally finite Cayley graph $\Gamma$ quasi-isometric to $\mathbb{H}^{3}$, are almost round. Given the graph $\Gamma$, for fixed $k$, there is a positive number $K$ such that all of the combinatorial disk-pairs $(D(R, n), D(R, n+k))$ are almost round $(K)$.

Proof of Theorem 4.2.1. This is Theorem 7.1 of [7], whose statement is due to Cannon and whose proof is due in part to Bestvina.

Proof of Theorem 4.2.2. This theorem was claimed by Cannon at the annual meeting of the American Mathematical Society in Phoenix, 1989. (See [6].) The first written proof was developed independently by Swenson [27]. We apply Zorn's Lemma to collections of the form

$$
\left\{\left(D\left(R_{i}, n\right), D\left(R_{i}, n+16 \delta\right)\right)\right\},
$$

where $D\left(R_{i}, n+16 \delta\right) \cap D\left(R_{j}, n+16 \delta\right)=\emptyset$ for $i \neq j$. By (3.23), a maximal element will be a pair-cover of $\partial \Gamma$.

Proof of Theorem 4.2.3. We need to compare nearest point projections in $\mathbb{H}^{3}$ and in $\Gamma$. The key to this comparison is that $\mathbb{H}^{3}$ and $\Gamma$ are quasi-isometric spaces of negative curvature (see [2], [5], [6], [10] for fundamental facts about quasi-isometries). Functions $f: X \rightarrow Y$ and $g: Y \rightarrow X$ between metric spaces $X$ and $Y$ are said to be approximately-inverse quasi-isometries $(\alpha, \beta)$ if

1. for each $x_{1}, x_{2} \in X, y_{1}, y_{2} \in Y$,

$$
\begin{gathered}
d\left(f\left(x_{1}\right), f\left(x_{2}\right)\right) \leq \alpha \cdot d\left(x_{1}, x_{2}\right)+\beta, \\
d\left(g\left(y_{1}\right), g\left(y_{2}\right)\right) \leq \alpha \cdot d\left(y_{1}, y_{2}\right)+\beta ;
\end{gathered}
$$

and

2. for each $x \in X, y \in Y$,

$$
d(g \circ f(x), x) \leq \beta \quad \text { and } \quad d(f \circ g(y), y) \leq \beta .
$$

By definition, $X$ and $Y$ are quasi-isometric if they admit approximately-inverse quasi-isometries $(\alpha, \beta)$ for some positive numbers $\alpha$ and $\beta$. By $[10,2.2]$ the quasiisometries induce homeomorphisms of the boundary in the case where $X$ and $Y$ are negatively curved. The following lemma supplies the key fact needed in the proof of Theorem 4.2.3. 
4.2.3.1. Lemma. Suppose $X$ and $Y$ are quasi-isometric spaces of negative curvature, that $f: X \rightarrow Y$ and $g: Y \rightarrow X$ are approximately-inverse quasi-isometries $(\alpha, \beta)$, and that $\delta$ is a thinness constant for both $X$ and $Y$. Then there is a constant $M>0$ depending only on $\alpha, \beta$, and $\delta$ such that nearest-point projections $p$ in $X$ and $q$ in $Y$ differ by at most $M$. That is, let $R:[0, \infty) \rightarrow X$ and $S:[0, \infty) \rightarrow Y$ denote rays such that $f \circ R(0)=S(0)$ and $f \circ R(\infty)=S(\infty)$. Let $p: X \rightarrow R$ and $q: Y \rightarrow S$ denote nearest point projections. Let $x \in X$. Then $d(f \circ p(x), q \circ f(x)) \leq M$.

Proof of Lemma 4.2.3.1. Construct the following diagram.
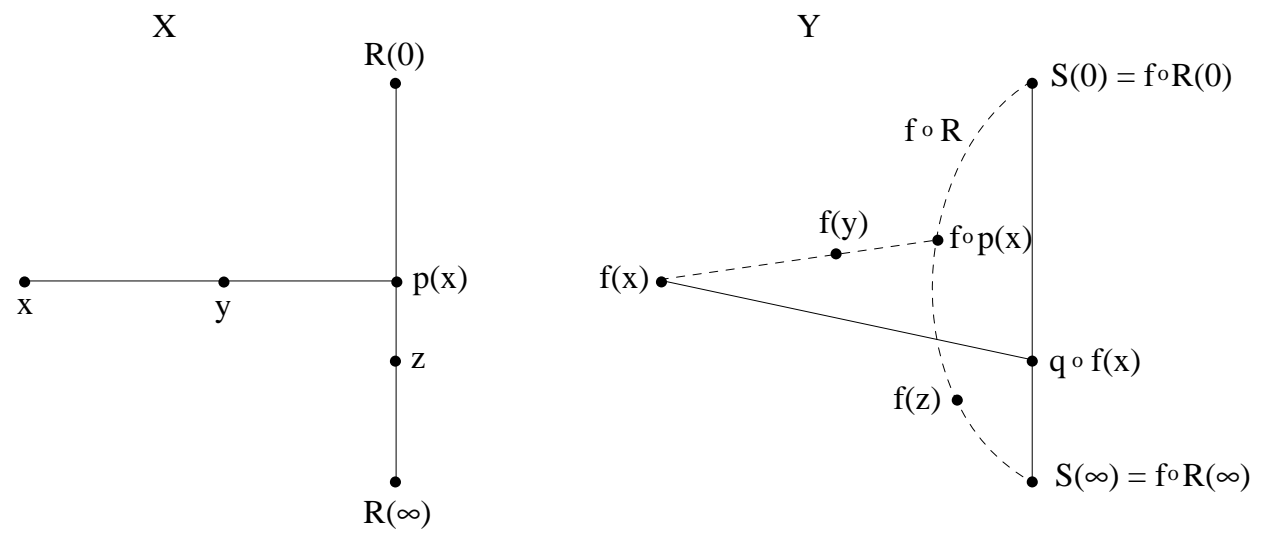

FiguRE 4.2.3.1

Solid lines denote geodesics. Dotted lines denote images of geodesics under $f$. Points $y$ and $z$ are chosen so that $f(y)$ and $f(z)$ are nearest point projections (or nearly so, since $f$ is not necessarily continuous so that the image of a compact set need not be closed) from $q \circ f(x)$ into $f([x, p(x)])$ and $f(R)$, respectively.

We verify in turn that

1. $d(q \circ f(x), f(y))$ and $d(q \circ f(x), f(z))$ are small;

2. $d(y, z)$ is small;

3. $d(y, p(x))$ is small;

4. $d(f(y), f \circ p(x))$ is small;

5. $d(q \circ f(x), f \circ p(x))$ is small, which is the conclusion of the lemma.

The one nonobvious fact used in these verifications is a property of quasi-isometries between negatively curved spaces which appears in [2], [5], [6], [10]: There is a constant $M^{\prime}$ depending only on $\alpha, \beta$, and $\delta$ such that the two sets $f([a, b])$ and $[f(a), f(b)]$ are everywhere within $M^{\prime}$ of one another. It follows immediately that $d(q \circ f(x), f(z)) \leq M^{\prime}$, which is one half of (1). To estimate $d(q \circ f(x), f(y))$, we consider the quadrilateral $\mathcal{P}[f(x), f \circ p(x), q \circ f \circ p(x), q \circ f(x)]$. By our nonobvious fact, $d(f \circ p(x), q \circ f \circ p(x)) \leq M^{\prime}$. By distance-from-projection,

$$
d(q \circ f(x),[f(x), f \circ p(x)] \cup[f \circ p(x), q \circ f \circ p(x)]) \leq 4 \delta .
$$

Hence

$$
d(q \circ f(x),[f(x), f \circ p(x)]) \leq 4 \delta+M^{\prime}
$$


and

$$
d(q \circ f(x), f(y))=d(q \circ f(x), f([x, p(x)])) \leq 4 \delta+2 M^{\prime} .
$$

These estimates complete the proof of (1).

Property (1) implies (2) since $f$ is a quasi-isometry, and property (2) implies property (3) since $d(y, p(x)) \leq d(y, z)$. Property (3) implies property (4) since $f$ is a quasi-isometry. Properties (1) and (4) when coupled by the triangle inequality imply property (5), and the proof of Lemma 4.2.3.1 is complete.

Proof of Theorem 4.2.3, continued. Let $f: \Gamma \rightarrow \mathbb{H}^{3}$ and $g: \mathbb{H}^{3} \rightarrow \Gamma$ be approximately-inverse quasi-isometries $(\alpha, \beta)$. Let $\delta$ be a common thinness constant for $\Gamma$ and $\mathbb{H}^{3}$. Let $R:[0, \infty) \rightarrow \Gamma$ be a ray. Let $S:[0, \infty) \rightarrow \mathbb{H}^{3}$ be a hyperbolic ray joining $f \circ R(0)$ to $f \circ R(\infty)$. Let $p: \Gamma \rightarrow R$ and $q: \mathbb{H}^{3} \rightarrow S$ denote nearest point projections. The function $q \circ f: R \rightarrow S$ is a quasi-isometry (see, for example, [2] or [10]), with constants depending only on $\alpha, \beta$, and $\delta$. The function $f \circ p$ approximates $q \circ f$ by Lemma 4.2.3.1. It follows easily from properties of quasiisometries that, if the nearest point projections $p(x)$ lie in the interval $R([n, n+k])$, then the projections $q \circ f(x)$ lie in an interval $S([m, m+l])$ with $l$ bounded in terms of $k$ (and $\alpha, \beta$, and $\delta$ ).

By the nonobvious fact of (4.2.3.1), any two rays $T \subset \Gamma$ and $T^{\prime} \subset \mathbb{H}^{3}$ with $f(T(0))=T^{\prime}(0)$ and $f(T(\infty))=T^{\prime}(\infty)$ have the property that $f(T)$ and $T^{\prime}$ are everywhere within $M^{\prime}+\delta$, as are $g\left(T^{\prime}\right)$ and $T$. Now, using (3.16) and (3.24),

$$
D(S, m+l+L) \subset D(R, n+k) \subset D(R, n) \subset D(S, m-L)
$$

for some $L$ depending only on $\alpha, \beta, \delta$, and the constants from (3.16) and (3.24).

Since the pair $(D(S, m-L), D(S, m+l+L))$ is geometrically almost round $(K)$ (whenever $m-L \geq 0$ ) as discussed in the opening paragraphs of 4.2 , so also is the combinatorial pair $(D(R, n), D(R, n+k))$ whenever $n$ is sufficiently large (independent of $R$ ).

4.3. Collections with bounded overlap. Two collections $\mathcal{D}$ and $\mathcal{D}^{\prime}$ of sets are said to have bounded overlap $(K)$ if no element of $\mathcal{D}$ intersects more than $K$ elements of $\mathcal{D}^{\prime}$, and no element of $\mathcal{D}^{\prime}$ intersects more than $K$ elements of $\mathcal{D}$.

4.3.1. Theorem. Covers with bounded overlap define comparable combinatorial moduli. Suppose $\mathcal{D}$ and $\mathcal{D}^{\prime}$ are finite covers of a ring $\mathcal{R}$ such that $\mathcal{D}$ and $\mathcal{D}^{\prime}$ have bounded overlap $(K)$. Then there is a constant $L$ depending only on $K$ such that the moduli $M$ and $m$ of $\mathcal{R}$ defined by $\mathcal{D}$ and $\mathcal{D}^{\prime}$ satisfy the inequalities

$$
M\left(\mathcal{R}, \mathcal{D}^{\prime}\right) \leq L \cdot M(\mathcal{R}, \mathcal{D}) \quad \text { and } \quad m\left(\mathcal{R}, \mathcal{D}^{\prime}\right) \leq L \cdot m(\mathcal{R}, \mathcal{D}) .
$$

In fact, we may take $L=K^{3}$.

4.3.2. Theorem. Restricted bounded overlap theorem. (A more general theorem appears in Section 5, Theorem 5.2.2.) Given $\Gamma$, a locally finite Cayley graph of a negatively curved group, there is a constant $K$ such that, for each $n, \mathcal{D}(n)$ has bounded overlap $(K)$ with itself.

Proof of Theorem 4.3.1. Let $\sigma^{\prime}$ and $\tau^{\prime}$ be weight functions for the covering $\mathcal{D}^{\prime}$ of the ring $\mathcal{R}$ which realize the two moduli, $M$ and $m$, so that

$$
M\left(\mathcal{R}, \mathcal{D}^{\prime}\right)=\frac{H^{2}\left(\mathcal{R}, \sigma^{\prime}\right)}{A\left(\mathcal{R}, \sigma^{\prime}\right)}
$$


and

$$
m\left(\mathcal{R}, \mathcal{D}^{\prime}\right)=\frac{A\left(\mathcal{R}, \tau^{\prime}\right)}{C^{2}\left(\mathcal{R}, \tau^{\prime}\right)} .
$$

Define weight functions $\sigma$ and $\tau$ on $\mathcal{D}$ as follows. If $d \in \mathcal{D}$, then

$$
\sigma(d)=\max \left\{\sigma^{\prime}\left(d^{\prime}\right) \mid d^{\prime} \in \mathcal{D}^{\prime}, d^{\prime} \cap d \neq \emptyset\right\}
$$

and

$$
\tau(d)=\max \left\{\tau^{\prime}\left(d^{\prime}\right) \mid d^{\prime} \in \mathcal{D}^{\prime}, d^{\prime} \cap d \neq \emptyset\right\} .
$$

It is convenient to assign choice functions $f, g: \mathcal{D} \rightarrow \mathcal{D}^{\prime}$ such that

$$
\sigma(d)=\sigma^{\prime}(f(d)) \text { and } d \cap f(d) \neq \emptyset,
$$

and

$$
\tau(d)=\tau^{\prime}(g(d)) \text { and } d \cap g(d) \neq \emptyset .
$$

We can then bound $\sigma$-areas as follows:

$$
A(\mathcal{R}, \sigma)=\sum_{d \in \mathcal{D}}(\sigma(d))^{2}=\sum_{d \in \mathcal{D}} \sigma^{\prime}(f(d))^{2} \leq \sum_{d^{\prime} \in \mathcal{D}^{\prime}} K \cdot \sigma^{\prime}\left(d^{\prime}\right)^{2}=K \cdot A\left(\mathcal{R}, \sigma^{\prime}\right) .
$$

Similarly, $A(\mathcal{R}, \tau) \leq K \cdot A\left(\mathcal{R}, \tau^{\prime}\right)$.

Let $\alpha$ be a path joining the ends of $\mathcal{R}$ such that the height $H(\mathcal{R}, \sigma)$ is equal to $L_{\sigma}(\alpha)$. We then have the following estimate.

$$
\begin{aligned}
H(\mathcal{R}, \sigma) & =L_{\sigma}(\alpha)=\sum\{\sigma(d) \mid d \in \mathcal{D}, d \cap \alpha \neq \emptyset\} \\
& =\sum\left\{\sigma^{\prime}(f(d)) \mid d \in \mathcal{D}, d \cap \alpha \neq \emptyset\right\} \\
& \geq \frac{1}{K} \sum\left\{\sigma^{\prime}\left(d^{\prime}\right) \mid d^{\prime} \in \mathcal{D}^{\prime}, d^{\prime} \cap \alpha \neq \emptyset\right\} \\
& =\frac{1}{K} L_{\sigma^{\prime}}(\alpha) \geq \frac{1}{K} \cdot H\left(\mathcal{R}, \sigma^{\prime}\right) .
\end{aligned}
$$

Similarly, $C(\mathcal{R}, \tau) \geq(1 / K) C\left(\mathcal{R}, \tau^{\prime}\right)$.

Hence we have

$$
M\left(\mathcal{R}, \mathcal{D}^{\prime}\right)=\frac{H^{2}\left(\mathcal{R}, \sigma^{\prime}\right)}{A\left(\mathcal{R}, \sigma^{\prime}\right)} \leq \frac{K^{2} H^{2}(\mathcal{R}, \sigma)}{(1 / K) A(\mathcal{R}, \sigma)} \leq K^{3} \cdot M(\mathcal{R}, \mathcal{D})
$$

and

$$
m\left(\mathcal{R}, \mathcal{D}^{\prime}\right)=\frac{A\left(\mathcal{R}, \tau^{\prime}\right)}{C^{2}\left(\mathcal{R}, \tau^{\prime}\right)} \geq(1 / K) \frac{A(\mathcal{R}, \tau)}{K^{2} C^{2}(\mathcal{R}, \tau)} \geq\left(1 / K^{3}\right) m(\mathcal{R}, \mathcal{D}) .
$$

Since the relationship between $\mathcal{D}$ and $\mathcal{D}^{\prime}$ is symmetric, the theorem follows.

Proof of Theorem 4.3.2. By (3.25), if $D(S, n)$ intersects $D(R, n)$, then we have $d(S(n), R(n)) \leq 12 \delta$. By $(3.21)$, the disk $D(S, n)$ is determined by a radial segment of length $\leq 8 \delta$. There are a bounded, finite number of radial segments of length $\leq 8 \delta$ within $12 \delta$ of $R(n)$. Theorem 4.3.2 follows.

Proof of Theorem 4.1. Since $\{D(R, n): n=1,2, \ldots\}$ is a basis at the point $R(\infty) \in$ $\partial \Gamma$ (see [27]), it follows by the compactness of $\partial \Gamma,(3.20)$, and (3.21) that the diameters of elements of $\mathcal{D}(n)$ go to zero uniformly as $n \rightarrow \infty$. By Theorems 4.2.2 and 4.2 .3 , for $n$ sufficently large, the cover $\mathcal{D}(n)$ can be reduced to a collection $\mathcal{D}^{\prime}(n)$ consisting of the large disks (outer disks) of an almost-round pair-cover of $\mathbf{S}^{2}$. Since Theorem 4.3.2 implies that $\mathcal{D}(n)$ has uniformly bounded overlap with 
itself, $\mathcal{D}(n)$ and $\mathcal{D}^{\prime}(n)$ also satisfy the same overlap condition with respect to each other. Hence, by Theorem 4.3.1, $\mathcal{D}(n)$ and $\mathcal{D}^{\prime}(n)$ define uniformly comparable combinatorial moduli on the rings of $\mathbf{S}^{2}$. But for $n$ sufficiently large, by Theorem 4.2.1, $\mathcal{D}^{\prime}(n)$ defines combinatorial moduli comparable with the analytic moduli of $\mathbf{S}^{2}$. Hence, for $n$ sufficiently large, $\mathcal{D}(n)$ defines moduli comparable with the analytic moduli of $\mathbf{S}^{2}$. Theorem 4.1 follows easily.

\section{IF THE COMBINATORIAL-DISK SEQUENCE IS CONFORMAL, THE GROUP HAS CONSTANT CURVATURE}

In this section we prove Theorem 5.1, which forms the second half of the main theorem.

We make the standard assumptions: $G$ is a negatively curved group with locallyfinite negatively-curved Cayley graph $\Gamma$, having thinness constant $\delta$ and space $\partial \Gamma$ at infinity equal to the 2 -sphere $\mathbf{S}^{2}$. We fix a base point $\mathcal{O}$ for rays and determine thereby a sequence of sets $\mathcal{D}(n)$ of combinatorial disks $D(R, n)$ for $R$ a ray based at $\mathcal{O}$ and $n$ a positive integer.

5.1. Theorem. Let $G$ denote a negatively curved group which has as its space at infinity the 2-sphere $\mathbf{S}^{2}$. The group $G$ has constant curvature if the sequence $\mathcal{D}(n)$ is conformal. That is, if the sequence $\mathcal{D}(n)$ is conformal, then $G$ acts isometrically, properly discontinuously, and cocompactly on $\mathbb{H}^{3}$.

The proof of Theorem 5.1 will appear in Subsection 5.3, after preparation. It is perhaps reasonable to conjecture even more.

5.1. Conjecture. If $G$ is a negatively curved group which has as its space at infinity the 2-sphere $\mathbf{S}^{2}$, then the group $G$ has constant curvature. That is, $G$ acts isometrically, properly discontinuously, and cocompactly on $\mathbb{H}^{3}$.

However, there are nontrivial difficulties involved in extending the theorem to the conjecture, as shown by the example in Section 8 .

5.2. Correlated geodesics and the general overlap theorem. We say that rays $R, R^{\prime}$ can be correlated if they are, at some point, no further than $4 \delta$ apart. In that case, we choose nonnegative integers $r, r^{\prime}$ such that $d\left(R(r), R^{\prime}\left(r^{\prime}\right)\right) \leq 4 \delta+1$ and say that $(R, r)$ and $\left(R^{\prime}, r^{\prime}\right)$ are correlated pairs; $r, r^{\prime}$ are called correlation $p a$ rameters.

Every pair $(S, s)$, where $S$ is a ray and $s$ a positive number, gives rise to an entire family of correlated pairs, which we say are correlated by $(S, s)$, as follows.

5.2.1. Correlation Lemma. If $R, R^{\prime}$ are rays such that $R(0), R^{\prime}(0) \in H^{c}(S, s)$ and $R(\infty), R^{\prime}(\infty) \in D(S, s+8 \delta)$, then $R$ and $R^{\prime}$ can be correlated. In fact, correlation parameters $r, r^{\prime}$ can be chosen to be integers such that

$$
d(R(r), S(s+4 \delta)), d\left(R^{\prime}\left(r^{\prime}\right), S(s+4 \delta)\right) \leq 2 \delta+1 / 2 .
$$

Proof of the Correlation Lemma. Since $R(0) \in H^{c}(S, s)$ and $R(\infty) \in D(S, s+8 \delta)$, $R$ must come within $2 \delta$ of $S(s+4 \delta)$ by (3.14), and similarly for $R^{\prime}$. A further move along $R, R^{\prime}$ of distance $\leq 1 / 2$ takes one to a vertex of $R, R^{\prime}$. The correlation lemma follows. 
5.2.2. General Overlap Theorem. Suppose the ray-point pair $(S, s)$ correlates pairs $(R, r)$ and $\left(R^{\prime}, r^{\prime}\right)$. If

$$
D(R, r+t) \cap D\left(R^{\prime}, r^{\prime}+t^{\prime}\right) \neq \emptyset,
$$

then, for $t, t^{\prime}$ sufficiently large (depending only on $\left.\delta\right), d\left(R(r+t), R^{\prime}\left(r^{\prime}+t^{\prime}\right)\right)$ can be bounded by a constant which depends only on $\left|t-t^{\prime}\right|$ and $\delta$.

Proof of the General Overlap Theorem. We need $t, t^{\prime}$ large with respect to $\delta$ and to $\left|t-t^{\prime}\right|$. Suppose

$$
D(R, r+t) \cap D\left(R^{\prime}, r^{\prime}+t^{\prime}\right) \neq \emptyset
$$

so that there exists $x \in H(R, r+t) \cap H\left(R^{\prime}, r^{\prime}+t^{\prime}\right)$. Construct the following diagram.

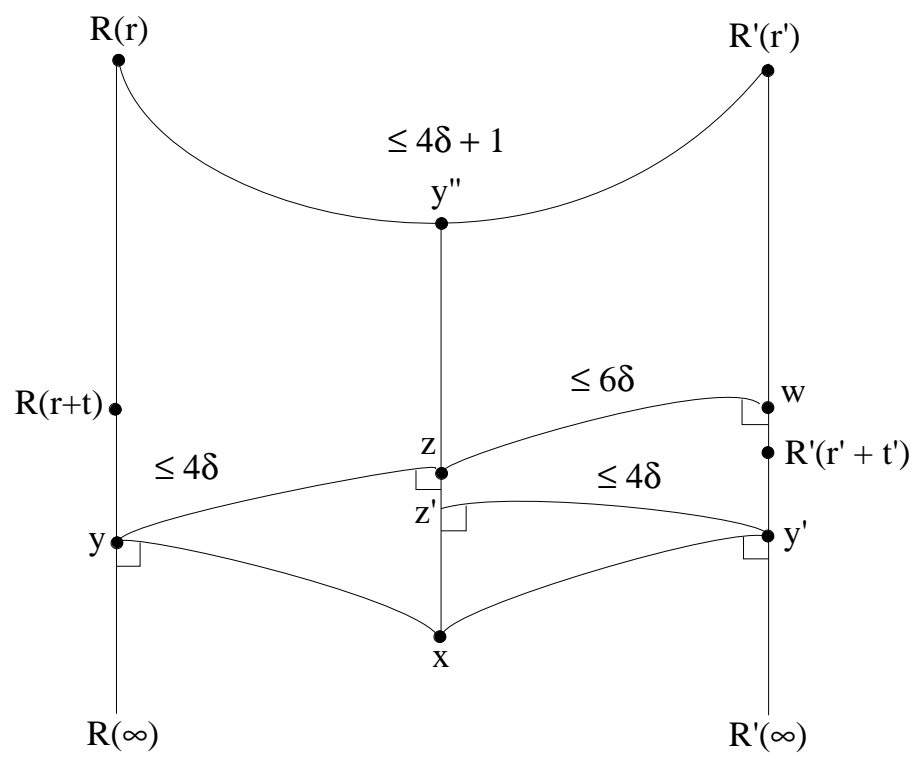

FiguRe 5.2.2.1

Points $y, y^{\prime}, y^{\prime \prime}$ are nearest point projections from $x$ into $R, R^{\prime}$, and $\left[R(r), R^{\prime}\left(r^{\prime}\right)\right]$, respectively, with $y \in[R(r+t), R(\infty)), y^{\prime} \in\left[R^{\prime}\left(r^{\prime}+t^{\prime}\right), R^{\prime}(\infty)\right)$. Points $z, z^{\prime}$ are nearest point projections from $y, y^{\prime}$ into $\left[x, y^{\prime \prime}\right]$, respectively. We lose no generality in assuming $z \in\left[y^{\prime \prime}, z^{\prime}\right]$. The point $w$ is the nearest point projection from $z$ into $\left[R^{\prime}\left(r^{\prime}\right), y^{\prime}\right]$.

Since $y$ is far from $\left[R(r), R^{\prime}\left(r^{\prime}\right)\right]$ ( $t$ and $t^{\prime}$ are large), by distance-from-projection, $d(y, z) \leq 4 \delta$. Similarly, $d\left(y^{\prime}, z^{\prime}\right) \leq 4 \delta$. Since $z$ is far from $\left[R(r), R^{\prime}\left(r^{\prime}\right)\right]\left(t\right.$ and $t^{\prime}$ are large), $z$ is within $2 \delta$ of $\left[R^{\prime}\left(r^{\prime}\right), y^{\prime}\right] \cup\left[y^{\prime}, z^{\prime}\right]$, hence within $6 \delta$ of $\left[R^{\prime}\left(r^{\prime}\right), y^{\prime}\right]$. Hence $d(y, w) \leq 10 \delta$. Since $R(r+t)$ is far from $\left[R(r), R^{\prime}\left(r^{\prime}\right)\right], R(r+t)$ is within $2 \delta$ of $\left[y, w, R^{\prime}\left(r^{\prime}\right)\right]$, hence within $12 \delta$ of $\left[R^{\prime}\left(r^{\prime}\right), w\right]$. Let $w^{\prime}$ be a nearest point projection of $R(r+t)$ in $\left[R^{\prime}\left(r^{\prime}\right), w\right]$. We then have Figure 5.2.2.2.

It follows easily that $w^{\prime}$ is within $16 \delta+1$ of $R^{\prime}\left(r^{\prime}+t\right)$. Hence $R(r+t)$ is within $12 \delta+(16 \delta+1)+\left|t-t^{\prime}\right|$ of $R^{\prime}\left(r^{\prime}+t^{\prime}\right)$. The proof is therefore complete. 


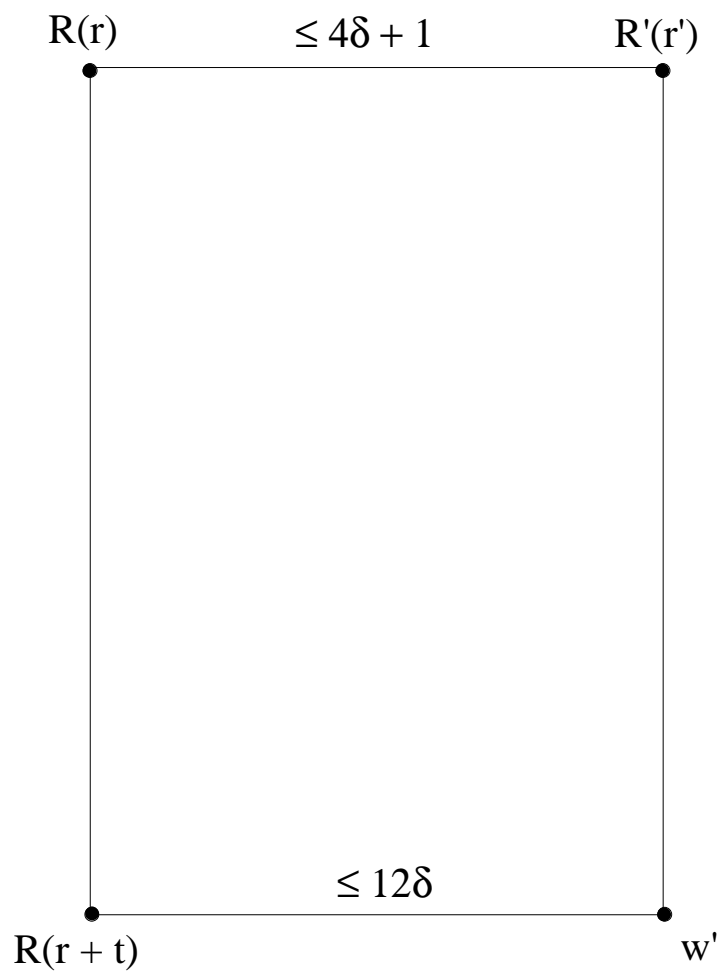

FiguRe 5.2.2.2

\subsection{Proof of Theorem 5.1.}

5.3.1. Theorem. Conformal sequences define analytic conformal structures. If the sequence $\mathcal{D}(n)$ is conformal, then it defines a quasiconformal structure on the 2 -sphere $\mathbf{S}^{2}$ quasiconformally equivalent with the standard analytic structure $\mathcal{S}$ on $\mathrm{S}^{2}$.

Proof of Theorem 5.3.1. By Theorem 4.3.2 applied in conjunction with (3.26), (3.27), and (3.28), the sequence $\mathcal{D}(n)$ has bounded overlap with a sequence $\mathcal{S}(n, m)$ of shinglings of $\mathbf{S}^{2}$. By Theorem 4.3.1, the sequence $\mathcal{S}(n, m)$ is also conformal. Hence, $\mathcal{S}(n, m)$ defines a quasiconformal structure on $\mathbf{S}^{2}$ by the Combinatorial Riemann Mapping Theorem [7].

By Theorem 6.2, any quasiconformal structure on $\mathbf{S}^{2}$ is quasiconformally equivalent with the standard analytic structure on $\mathbf{S}^{2}$. (We have enough extra conditions in our situation that we could apply Theorem 6.3 instead.)

5.3.2. Lemma. The group action is uniformly quasiconformal. With respect to the analytic conformal structure on $\mathbf{S}^{2}$ whose existence is asserted by Theorem 5.3.1, the action of $G$ on $\mathbf{S}^{2}$ is uniformly quasiconformal.

Proof. Theorem 5.3.1 asserts the existence of an analytic structure $\mathcal{S}$, which makes $\partial \Gamma=\mathbf{S}^{2}$ into a round 2 -sphere, such that the combinatorial moduli defined by the sequence $\mathcal{D}(n)$ are uniformly comparable to the analytic moduli defined by $\mathcal{S}$. 
Our immediate goal is to show that the action of $G$ on $\mathbf{S}^{2}=\partial \Gamma$ induced by left multiplication is uniformly quasiconformal in the analytic sense with respect to the structure $\mathcal{S}$.

We prove analytic quasiconformality by proving combinatorial quasiconformality in the small. The combinatorial effect of left multiplication by a group element of $G$ is change of base point from $\mathcal{O}$ to another vertex $\mathcal{O}^{\prime}$. This left multiplication induces a homeomorphism $f: \Gamma \cup \partial \Gamma \rightarrow \Gamma \cup \partial \Gamma$ which takes the sequence $\mathcal{D}(n)$ to the sequence $\mathcal{D}^{\prime}(n)$. Consequently a ring $\mathcal{Q}$ of $\mathcal{D}(n)$-modulus $M$ is taken to a ring $\mathcal{R}$ of $\mathcal{D}^{\prime}(n)$-modulus $M$. Combinatorial quasiconformality therefore requires that the $\mathcal{D}^{\prime}(n)$-modulus of $\mathcal{R}$ be uniformly comparable with the $\mathcal{D}(n)$-modulus of $\mathcal{R}$. For small rings $\mathcal{R}$ we shall establish this comparability by means of the general overlap theorem, Theorem 5.2.2. Since combinatorial moduli and analytic moduli are comparable, it follows that $f$ preserves analytic moduli of small rings within a constant multiple. But since analytic quasiconformality need only be checked for small rings, we deduce that $f$ is analytically quasiconformal as required, with the coefficient of quasiconformality independent of the group element.

Here is the proof that, for small rings $\mathcal{R}$, the $\mathcal{D}(n)$-modulus and the $\mathcal{D}^{\prime}(n)$ modulus are uniformly comparable. We define 'small' by means of pairs $(S, s)$ where $S$ is a ray and $s$ is a positive number such that $\mathcal{O}, \mathcal{O}^{\prime} \in H^{c}(S, s)$. We say that $\mathcal{R}$ is small if $\mathcal{R} \subset D(S, s+8 \delta)$.

Using the fact that the diameters of elements of $\mathcal{D}(n), \mathcal{D}^{\prime}(n)$ go to zero uniformly as $n \rightarrow \infty$, we may fix integers $m$ and $m^{\prime}$ so large that, if

$$
D(R, m) \cap \mathcal{R} \neq \emptyset, R(0)=\mathcal{O}, D\left(R^{\prime}, m^{\prime}\right) \cap \mathcal{R} \neq \emptyset, R^{\prime}(0)=\mathcal{O}^{\prime},
$$

then $R(\infty), R^{\prime}(\infty) \in D(S, s+8 \delta)$. We require further of $m$ and $m^{\prime}$ that $m-$ $d(\mathcal{O}, S(s+4 \delta))$ and $m^{\prime}-d\left(\mathcal{O}^{\prime}, S(s+4 \delta)\right)$ differ by at most 1 . We then let $\mathcal{E}(\mathcal{R}, m)$ and $\mathcal{E}^{\prime}\left(\mathcal{R}, m^{\prime}\right)$ denote the subcollections of $\mathcal{D}(m)$ and $\mathcal{D}^{\prime}\left(m^{\prime}\right)$ intersecting $\mathcal{R}$. Suppose $D(R, m) \in \mathcal{E}(\mathcal{R}, m)$ and $D\left(R^{\prime}, m^{\prime}\right) \in \mathcal{E}^{\prime}\left(\mathcal{R}, m^{\prime}\right)$ intersect. Then $R(0), R^{\prime}(0)=\mathcal{O}, \mathcal{O}^{\prime}$ $\in H^{c}(S, s)$ and $R(\infty), R^{\prime}(\infty) \in D(S, s+8 \delta)$, so that $R$ and $R^{\prime}$ are correlated by $(S, s)$, say with correlated pairs $(R, r)$ and $\left(R^{\prime}, r^{\prime}\right)$. Let $m=r+t$ and $m^{\prime}=r^{\prime}+t^{\prime}$; we require that $m, m^{\prime}$ be large enough so that $t, t^{\prime}$ satisfy the conditions of Theorem 5.2.2. Then it is easy to see that $\left|t-t^{\prime}\right|$ is uniformly bounded. By the general overlap theorem, Theorem 5.2.2, there is a uniform bound on $d\left(R(m), R^{\prime}\left(m^{\prime}\right)\right)$. Consequently there is a uniform bound on the number of elements of $\mathcal{E}^{\prime}\left(\mathcal{R}, m^{\prime}\right)$ intersecting a given element of $\mathcal{E}(\mathcal{R}, m)$, and vice versa. By Theorem 4.3.1, $\mathcal{E}(\mathcal{R}, m)$ and $\mathcal{E}^{\prime}\left(\mathcal{R}, m^{\prime}\right)$ define comparable moduli on $\mathcal{R}$. But these moduli are those defined by $\mathcal{D}$ and $\mathcal{D}^{\prime}$. We conclude that left multiplication is uniformly quasiconformal.

Proof of Theorem 4.2. In view of Theorem 5.3.1 and Lemma 5.3.2, Theorem 7.1 applies, so that Theorem 7.1 will complete the proof.

\section{QUASICONFORMAL SURFACES}

6.1. Definition. A surface $S$ is a connected metrizable 2-manifold without boundary. A $K$-quasiconformal structure on $S$ consists of an open cover $\mathcal{U}$ of $S$ and, for each element $u \in \mathcal{U}$, a homeomorphism $h_{u}$ from $u$ onto an open subset of the complex plane $\mathbf{C}$ such that the transition functions

$$
h_{v} h_{u}^{-1}: h_{u}(u \cap v) \rightarrow h_{v}(u \cap v)
$$


are $K$-quasiconformal for each $u, v \in \mathcal{U}$. The elements of the open cover $\mathcal{U}$ are called charts.

6.2. Theorem. ([17]) A compact surface $S$ with a quasiconformal structure is quasiconformally equivalent to some Riemann surface. Consequently the universal cover of $S$ is quasiconformally equivalent with one of the three standard simply connected Riemann surfaces, namely, the Riemann sphere, the complex plane, or the open unit disk.

Proof. The review of [17] states: "The author introduces the concept of quasiconformal manifold. His main result is that quasiconformal manifolds are essentially the same as Riemann surfaces."

We outline a proof on the basis of the information appearing in [19], but the proof is rather long and will not be included here. The following are the main steps: Step 1. The surface $S$ is homeomorphic with a polyhedral surface $S^{\prime}$ (standard topological fact). The resulting topological triangulation of $S$ may be chosen with simplexes so small that each lies in a single chart $u \in \mathcal{U}$ defining the quasiconformal structure on $S$. Step 2. The 1-skeleton may be modified so that the boundary of each 2-simplex is a quasiconformal simple closed curve. Step 3. One may construct a homeomorphism from the 1-skeleton of $S^{\prime}$ to the 1-skeleton of $S$ in such a way that, restricted to the boundary of any 2 -simplex, the homeomorphism is quasisymmetric. Step 4 . Since the map on the boundary of any 2 -simplex is quasisymmetric, the map may be extended to a quasiconformal homeomorphism on that 2-simplex. The resulting homeomorphism $f: S^{\prime} \rightarrow S$ is quasiconformal. Step 5. By a standard argument (see, for example, [24]), the polyhedron $S^{\prime}$ is quasiconformally equivalent to a Riemann surface $S^{\prime \prime}$, and the proof is complete.

Here is an alternative theorem which proves a special case sufficient for the purposes of the main theorem.

6.3. Theorem. Suppose $S$ is a topological 2-sphere with a quasiconformal atlas $(\mathcal{U}, f)$, where $\mathcal{U}$ has just two elements $u$ and $v$, each an open disk. Then there is a quasiconformal homeomorphism from $S$ onto the extended complex plane $\hat{\mathbf{C}}=$ $\mathbf{C} \cup\{\infty\}$ with its standard (quasi) conformal structure.

Proof. By standard topological arguments, there is a simple closed curve $J$ in $u \cap v$ such that the two disks $D_{1}$ and $D_{2}$ in $S$ bounded by $J$ lie in $u$ and in $v$, respectively. We may adjust $J$ slightly so that the image $J_{u}$ of $J$ under $f_{u}$ is a polygonal curve in $f_{u}(u) \subset \mathbf{C}$.

Recall [19, II.8.2, p. 97] that an arc or simple closed curve $A$ in $\mathbf{C}$ is quasiconformal if there exists a quasiconformal mapping of a domain $G \supset A$ which carries $A$ into a line or circle in $\mathbf{C}$. The property of being quasiconformal is a local property [19, II.8.9], and this local property is obviously invariant under quasiconformal homeomorphisms. Consequently, since $J_{u}$, as a polygonal curve in $f_{u}(u) \subset \mathbf{C}$, is obviously locally quasiconformal (one bend can be straightened by a single quasiconformal map), both $J_{u}$ and $J_{v}=f_{v}(J)$ are quasiconformal curves in C.

By [19, II.8.2, p. 97], there are quasiconformal homeomorphisms of the entire extended complex plane $\hat{\mathbf{C}}$ taking $J_{u}$ and $J_{v}$ to round circles in $\mathbf{C}$. Composing these mappings with the original mappings $f_{u}$ and $f_{v}$, we abandon the original mappings $f_{u}$ and $f_{v}$ and curves for the composites and new images so that the new $J_{u}$ and $J_{v}$ are round circles in $\hat{\mathbf{C}}$. 
There is a circle $J_{u}^{\prime}$ concentric with the circle $J_{u}$ in the disk $D_{u}=f_{u}\left(D_{1}\right)$ such that the annulus or ring $\mathcal{R}$ bounded by $J_{u}$ and $J_{u}^{\prime}$ in $D_{u}$ lies in $f_{u}(u \cap v)$. The transition function $f_{v} \circ f_{u}^{-1}$ takes $\mathcal{R}$ to a topological ring $\mathcal{R}^{\prime}$ which, except for the curve $J_{v}=f_{v} \circ f_{u}^{-1}\left(J_{u}\right)$, lies outside the circle $J_{v}$ in C. By [19, II.8.3, Theorem 8.3] this map on $\mathcal{R}$ can be extended to take $D_{u}$ quasiconformally onto the disk in the extended complex plane $\hat{\mathbf{C}}$ formed by adding the point at infinity to the domain outside the circle $J_{v}$. Let $f^{\prime}: D_{u} \rightarrow \hat{\mathbf{C}}$ be the resulting embedding.

Finally, we define $h: S \rightarrow \hat{\mathbf{C}}$ piecewise by the formulas

$$
h(x)=f^{\prime} \circ f_{u}(x) \text { if } x \in D_{1}
$$

and

$$
h(x)=f_{v}(x) \text { if } x \in D_{2} .
$$

As a composite of quasiconformal mappings, $h$ is clearly quasiconformal on int $D_{1}$ and int $D_{2}$. On a neighborhood of $J, h$ coincides with $f_{v}$. Hence $h$ is everywhere quasiconformal.

\section{Quasiconformal Groups aCting ON $\mathbf{S}^{2}$}

Theorem 7.1 was used in Section 5 to complete the proof of the main theorem.

7.1. Theorem. Suppose $G$ is a negatively curved group with locally-finite Cayley graph $\Gamma$. If $\partial \Gamma=\mathbf{S}^{2}$, and if the action of $G$ on $\mathbf{S}^{2}$ is uniformly quasiconformal with respect to the standard conformal structure on $\mathbf{S}^{2}$, then

1. the action is quasiconformally conjugate to a conformal action on $\mathbf{S}^{2}$; and

2. the conformal action on $\mathbf{S}^{2}$ induces an action on hyperbolic 3 -space $\mathbb{H}^{3}$ which is isometric, properly discontinuous, and cocompact.

The conclusions of the proof will be dealt with one at a time in the subsections of Section 7.

7.2. The action is conjugate to a conformal action. Conclusion (1) is one of the main conclusions of both [26] and [30]. Therefore we may assume that the action of $G$ on $\mathbf{S}^{2}$ is conformal.

7.3. Isometry. For standard properties of hyperbolic space and its relationship with the Riemann sphere and conformal mappings, see, for example, [3], [18], [21], [25]. The only conformal homeomorphisms of the Riemann sphere $\mathbf{S}^{2}$ are the linear or conjugate linear fractional transformations. These transformations are precisely the isometries of $\mathbb{H}^{3}$. Hence the induced action of $G$ on $\mathbb{H}^{3}$ is isometric.

7.4. Proper discontinuity. Proper discontinuity of $G$ on $\mathbb{H}^{3}$ is equivalent to the discreteness of the action of $G$ on $\mathbf{S}^{2}$ (see, for example, [18] for precise references to the proof).

7.4.1. Lemma [30]. A sufficient condition that $G$ acting on $\mathbf{S}^{2}$ be discrete in the compact open topology on Homeo $\left(\mathbf{S}^{2}\right)$ is that $G$ act properly discontinuously on the triple space $\mathcal{T}$ (definition recalled below) of $\mathbf{S}^{2}$.

Definition. Consider the action

$$
\left(\Sigma_{3}\right) \times\left(\mathbf{S}^{2} \times \mathbf{S}^{2} \times \mathbf{S}^{2}\right) \rightarrow\left(\mathbf{S}^{2} \times \mathbf{S}^{2} \times \mathbf{S}^{2}\right),
$$


where $\Sigma_{3}$ is the symmetric group on three letters which acts by permutation of coordinates. The triple space $\mathcal{T}$ is the subspace of the orbit space consisting of those orbits represented by triples

$$
\left(x_{1}, x_{2}, x_{3}\right) \in \mathbf{S}^{2} \times \mathbf{S}^{2} \times \mathbf{S}^{2}
$$

with $x_{1}, x_{2}$, and $x_{3}$ distinct. We denote the orbit by $\left[x_{1}, x_{2}, x_{3}\right]$. A basic open set is the orbit of a product open set $U_{1} \times U_{2} \times U_{3}$, with $U_{1}, U_{2}, U_{3}$ pairwise disjoint, $x_{1} \in U_{1}, x_{2} \in U_{2}, x_{3} \in U_{3}$.

Proof of Lemma 7.4.1. The compact open topology on the space Homeo $\left(\mathbf{S}^{2}\right)$ makes this space separable metric. Therefore, if $G$ is not discrete in this topology, there exist elements $g_{1}, g_{2}, \ldots$ converging to $g \in G$. Thus $h_{1}=g_{1} g^{-1}, h_{2}=g_{2} g^{-1}, \ldots$ converge to $1 \in G$. Let $\left[x_{1}, x_{2}, x_{3}\right] \in \mathcal{T}$. Let $C_{1}, C_{2}, C_{3}$ denote pairwise disjoint compact neighborhoods of $x_{1}, x_{2}, x_{3}$ in $\mathbf{S}^{2}$. Then the orbit $\left[C_{1} \times C_{2} \times C_{3}\right]$ of $C_{1} \times C_{2} \times C_{3}$ under $\sigma_{3}$ is a compact neighborhood of $\left[x_{1}, x_{2}, x_{3}\right]$ in $\mathcal{T}$. But, for $i$ sufficiently large, $h_{i}\left(\left[x_{1}, x_{2}, x_{3}\right]\right) \in\left[C_{1} \times C_{2} \times C_{3}\right]$. Thus $G$ does not act properly discontinuously on $\mathcal{T}$.

We now prove that $G$ does indeed act properly discontinuously on the triple space of $\mathbf{S}^{2}$. First we need the following lemma.

7.4.2. Lemma. Let $R, S$, and $T$ denote inequivalent rays in the Cayley graph $\Gamma$. Then there exist half-spaces $H(R, r), H(S, s), H(T, t)$ such that, if $\alpha, \beta, \gamma$ are geodesic arcs joining these half-spaces in pairs, then there is a point $b$ near to all three. If $\alpha^{\prime}, \beta^{\prime}, \gamma^{\prime}$ also join the half spaces and $b^{\prime}$ is near all three, then $b$ and $b^{\prime}$ are near one another. We call $b$ an approximate barycenter for $R(\infty), S(\infty)$, $T(\infty)$.

Proof. We construct the following diagram.

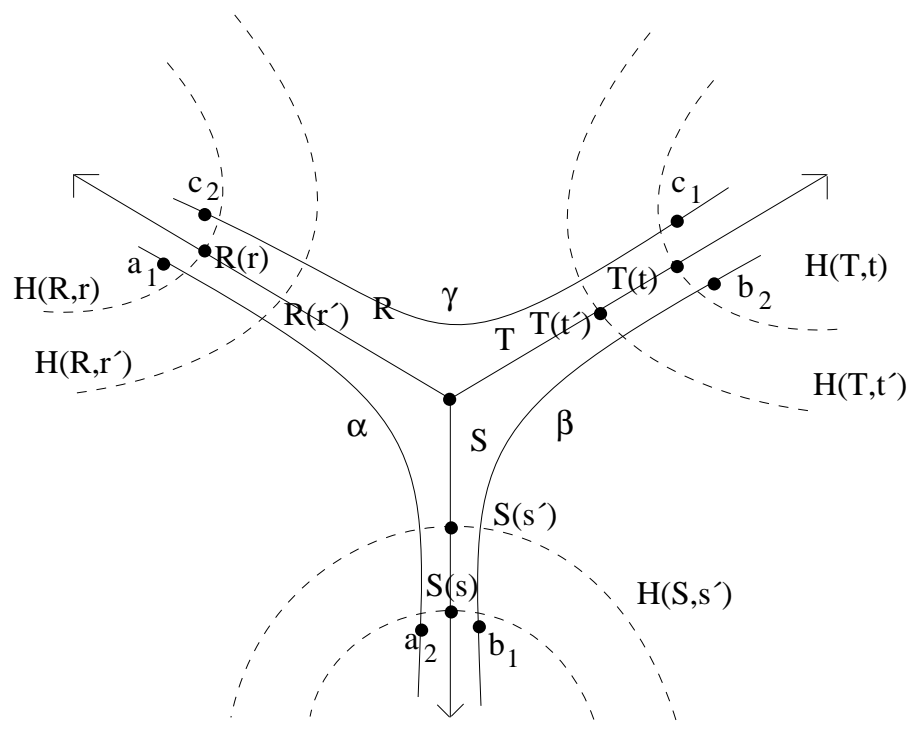

$\mathrm{H}(\mathrm{S}, \mathrm{s})$

FiguRe 7.4.2.1 
We have buffered $H(R, r)$ by $H\left(R, r^{\prime}\right), H(S, s)$ by $H\left(S, s^{\prime}\right), H(T, t)$ by $H\left(T, t^{\prime}\right)$. Using (3.17), we assume $r^{\prime}, s^{\prime}, t^{\prime}$ so large that no $\alpha$ can penetrate into $H\left(T, t^{\prime}\right)$, no $\beta$ into $H\left(R, r^{\prime}\right)$, no $\gamma$ into $H\left(S, s^{\prime}\right)$; in fact, by (3.16), so large that $H\left(R, r^{\prime}\right)$ must be far from $\beta, H\left(S, s^{\prime}\right)$ from $\gamma$, and $H\left(T, t^{\prime}\right)$ from $\alpha$. We assume $r-r^{\prime}, s-s^{\prime}$, and $t-t^{\prime}$ also large. These choices implicitly restrict the half-spaces $H(R, r), H(S, s)$, and $H(T, t)$, and there certainly exist such half-spaces. We show that such half-spaces satisfy the requirements of the lemma.

Let $\alpha, \beta, \gamma$ be given. The arcs $\alpha$ and $\gamma$ can be joined by a small arc $\left[c_{2}, a_{1}\right]$ near $R(r)$ by (3.14), $\alpha$ and $\beta$ by a small arc $\left[a_{2}, b_{1}\right]$ near $S(s), \beta$ and $\gamma$ by a small arc $\left[b_{2}, c_{1}\right]$ near $T(t)$. The hexagon $\mathcal{P}\left[a_{1}, a_{2}, b_{1}, b_{2}, c_{1}, c_{2}\right]$ is $4 \delta$-thin. Let $\alpha_{0}$ be a subarc of $\alpha$ joining $H\left(R, r^{\prime}\right)$ to $H\left(S, s^{\prime}\right)$ irreducibly. Since $r-r^{\prime}, s-s^{\prime}, t-t^{\prime}$ are all large, no point of $\alpha_{0}$ can be near any one of $\left[c_{2}, a_{1}\right],\left[a_{2}, b_{1}\right],\left[b_{2}, c_{1}\right]$. Hence every point of $\alpha_{0}$ must lie within $4 \delta$ of one of $\left[b_{1}, b_{2}\right]$ and $\left[c_{1}, c_{2}\right]$. Points of $\alpha_{0}$ near $R\left(r^{\prime}\right)$ are far from $\beta$. Points of $\alpha_{0}$ near $S\left(s^{\prime}\right)$ are far from $\gamma$. Hence some points of $\alpha_{0}$ are within $4 \delta$ of $\left[b_{1}, b_{2}\right]$, some within $4 \delta$ of $\left[c_{1}, c_{2}\right]$. We conclude that some point $b$ of $\alpha_{0}$ is within $4 \delta$ of both $\left[b_{1}, b_{2}\right]$ and $\left[c_{1}, c_{2}\right]$. Note that since $H\left(R, r^{\prime}\right)$ is far from $\gamma, b$ must be well outside $H\left(R, r^{\prime}\right)$. Similarly, $b$ must be far from $H\left(S, s^{\prime}\right)$ and $H\left(T, t^{\prime}\right)$.

Now let other $\operatorname{arcs} \alpha^{\prime}, \beta^{\prime}, \gamma^{\prime}$, with associated point $b^{\prime}$, be given. We need to show that $b$ and $b^{\prime}$ are near one another. Since $\alpha$ and $\alpha^{\prime}$ both come near $R(r)$ and near $S(s)$, long portions of $\alpha$ and $\alpha^{\prime}$ must have nearby endpoints so that those entire portions are near one another by (3.5). It follows that $b^{\prime}$ is near $\alpha$ as well as near $\alpha^{\prime}$. Similarly, $b^{\prime}$ is near $\beta$ and $\gamma$. Thus we have the following diagram.

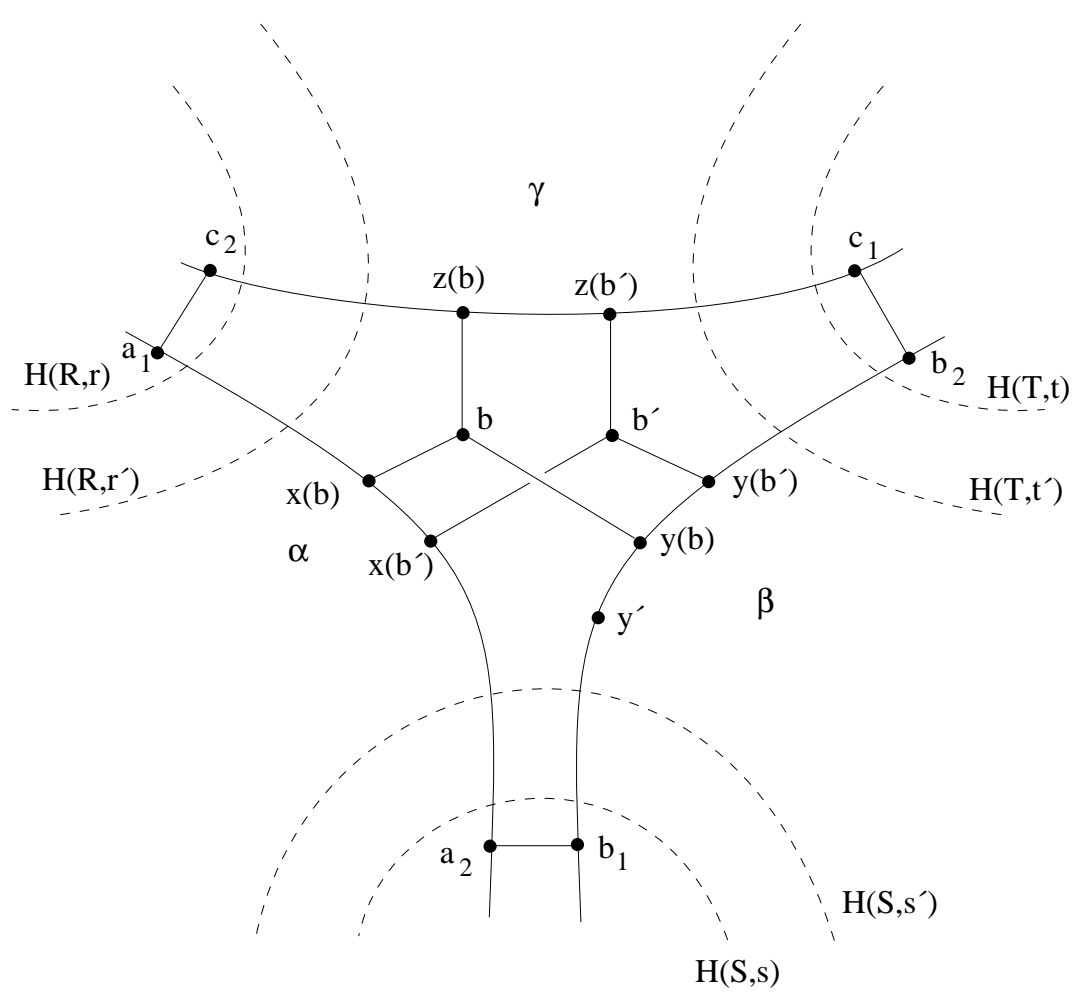

Figure 7.4.2.2 
The $\operatorname{arcs}[b, x(b)],[b, y(b)],[b, z(b)],\left[b^{\prime}, x\left(b^{\prime}\right)\right],\left[b^{\prime}, y\left(b^{\prime}\right)\right],\left[b^{\prime}, z\left(b^{\prime}\right)\right]$ are all assumed small and, as noted before, must be far from $H\left(R, r^{\prime}\right), H\left(S, s^{\prime}\right), H\left(T, t^{\prime}\right)$. Simple enumeration of cases shows that we lose no generality in assuming $x(b), x\left(b^{\prime}\right), y(b)$, $y\left(b^{\prime}\right)$ ordered as shown on $\alpha$ and $\beta$; that is, we may assume $x\left(b^{\prime}\right)$ between $x(b)$ and $a_{2}, y(b)$ between $y\left(b^{\prime}\right)$ and $b_{1}$ (we may have to permute $R, S, T$ to attain this condition).

We are now ready to show that $d\left(b, b^{\prime}\right)$ is small by showing $d\left(y(b), y\left(b^{\prime}\right)\right)$ is small. The two arcs $\left[x(b), a_{2}\right]$ and $\left[y(b), b_{1}\right]$ have their endpoints near one another; hence they are everywhere near by (3.5). Hence $x\left(b^{\prime}\right)$ is near some $y^{\prime}$ in $\left[y(b), b_{1}\right]$. Thus $y\left(b^{\prime}\right)$ is near $b^{\prime}$, which is near $x\left(b^{\prime}\right)$, which is near $y^{\prime}$. But $d\left(y(b), y\left(b^{\prime}\right)\right) \leq d\left(y^{\prime}, y\left(b^{\prime}\right)\right)$, and we are done.

7.4.3. Theorem (Freden). Let $G$ be a negatively curved group, $\Gamma$ a locally-finite Cayley graph for $G, \partial \Gamma$ the space at infinity, and $\mathcal{T}$ the triple space associated with $\partial \Gamma$. Then $G$ acts properly discontinuously on $\mathcal{T}$.

Proof. Suppose $G$ does not act properly discontinuously on $\mathcal{T}$. Let $C$ be a compact subset of $\mathcal{T}$ and $g_{1}, g_{2}, \ldots \in G$ be such that $g_{i}(C) \cap C \neq \emptyset$ for each $i$. Let $x_{i}=\left[x_{i 1}, x_{i 2}, x_{i 3}\right]$ be an element of $C$ such that

$$
g_{i}\left(x_{i}\right)=y_{i}=\left[y_{i 1}, y_{i 2}, y_{i 3}\right] \in C .
$$

Without loss of generality, $x_{i}$ converges to $X=\left[X_{1}, X_{2}, X_{3}\right] \in \mathcal{T}$ and $y_{i}$ converges to $Y=\left[Y_{1}, Y_{2}, Y_{3}\right] \in \mathcal{T}$. Let $R_{1}, R_{2}, R_{3}$ be rays from the base point $\mathcal{O}$ to $X_{1}, X_{2}$, $X_{3}$. Let $S_{1}, S_{2}, S_{3}$ be rays from $\mathcal{O}$ to $Y_{1}, Y_{2}, Y_{3}$. Pick $t$ so large that if $\alpha, \beta, \gamma$ are geodesic arcs joining the half-spaces $H\left(R_{1}, t\right), H\left(R_{2}, t\right), H\left(R_{3}, t\right)$ in pairs, the conclusions of Lemma 7.4.2 are satisfied, and similarly for $\operatorname{arcs} \alpha^{\prime}, \beta^{\prime}, \gamma^{\prime}$ joining half-spaces $H\left(S_{1}, t\right), H\left(S_{2}, t\right), H\left(S_{3}, t\right)$ in pairs. Hence using (3.12) there exist approximate barycenters $b_{i}$ for all the triples $x_{i}, i$ sufficiently large, with $b_{i}$ near an approximate barycenter $b$ for $X$. Similarly, $g_{i}\left(b_{i}\right)$ is an approximate barycenter for $y_{i}$ which, for large $i$, is near a barycenter $b^{\prime}$ for $Y$. Hence $g_{i}(b)$ must also be near $b^{\prime}$ since

$$
d\left(g_{i}(b), g_{i}\left(b_{i}\right)\right)=d\left(b, b_{i}\right) .
$$

Thus the points $g_{i}(b)$ stay in a compact set of $\Gamma$. But $G$ acts properly discontinuously on $\Gamma$, a contradiction.

From Lemma 7.4.1 and Theorem 7.4.3 we see that the action of $G$ on $\mathbb{H}^{3}$ is properly discontinuous.

7.5. Cocompactness. Eric Freden has proved that every point of $\mathbf{S}^{2}$ is a point of approximation of $G$ :

7.5.1. Theorem (Freden). Let $G$ be a negatively curved group, $\Gamma$ a locally-finite Cayley graph for $G, \partial \Gamma$ the space at infinity. Then every point of $\partial \Gamma$ is a point of approximation (definition reviewed below) for the action of $G$ on $\partial \Gamma$.

7.5.2. Definition. A point $p$ of $\partial \Gamma$ is a point of approximation for the action of $G$ on $\partial \Gamma$ if there is a sequence $g_{1}, g_{2}, \ldots$ of distinct elements of $G$ and a positive number $\alpha$ so that, for each compact subset $C \subset \partial \Gamma \backslash\{p\}$ and for all sufficiently large $i, d\left(g_{i}(p), g_{i}(C)\right) \geq \alpha$. (See [21, VI.B.1].)

Proof of Theorem 7.5.1. Choose a ray $R:[0, \infty) \rightarrow \Gamma$ such that $R(0)=i d \in G$ and $R(\infty)=p$. Let $g_{n}$ be the element of $G$ which by left multiplication on $\Gamma$ takes 
$R(n)$ to $i d \in G$. Choose a subsequence $g_{n 1}, g_{n 2}, \ldots$ of $g_{1}, g_{2}, \ldots$ such that the image rays $g_{n i} \circ R([0, \infty))$ converge setwise to a line $L:(-\infty, \infty) \rightarrow \Gamma, L(0)=i d$. We can describe $L$ very precisely. Let $A_{n}:(-\infty, \infty) \rightarrow(-\infty, \infty)$ be the shift map, $x \mapsto x+n$. The function $f_{n i}=g_{n i} \circ R \circ A_{n i}:\left[-n_{i}, 0\right] \rightarrow \Gamma$ is a segment with $f_{n i}(0)=i d \in G$. The segments $f_{n i}$ converge to $L \mid(-\infty, 0]$. The function $h_{n i}=g_{n i} \circ R \circ A_{n i}:[0, \infty) \rightarrow \Gamma$ is a ray with $h_{n i}(0)=i d$. The rays $h_{n i}$ converge to $L \mid[0, \infty)$. Now let $i$ be very large. Then $g_{n i} \circ R([0, \infty))$ and $L((-\infty, \infty))$ will share a very large segment centered at $i d$. Pick a large integer $m_{i}$ such that

$$
0 \ll n_{i}-m_{i}<n_{i}<n_{i}+m_{i}
$$

and such that the points $L\left(-m_{i}\right)=g_{n i} \circ R\left(n_{i}-m_{i}\right)$, id $=L(0)=g_{n i} \circ R\left(n_{i}\right)$, and $L\left(m_{i}\right)=g_{n i} \circ R\left(n_{i}+m_{i}\right)$ all lie well within the segment of intersection. Then $D\left(R, n_{i}-m_{i}\right)$ and $D\left(R, n_{i}+m_{i}\right)$ will be very small neighborhoods of $p$ in $\partial \Gamma$. The image $g_{n i}\left(D\left(R, n_{i}+m_{i}\right)\right)=D\left(L, m_{i}\right)$ will be a very small neighborhood of $L(\infty)$, showing that $g_{n i}$ maps $p$ very near to $L(\infty)$. The image $g_{n i}\left(\partial \Gamma \backslash D\left(R, n_{i}-m_{i}\right)\right)=$ $\partial \Gamma \backslash D\left(L,-m_{i}\right)$ will be a very small neighborhood of $L(-\infty)$, which shows that $g_{n i}$ maps almost all of $\partial \Gamma \backslash\{p\}$ very near to $L(-\infty)$. We conclude that $p$ is a point of approximation, as claimed.

Since every point of $\mathbf{S}^{2}$ is a point of approximation, $G$ is geometrically finite by [21, VI.C.7] and has compact fundamental polyhedron by [21, VI.B.5]. Therefore the action of $G$ on $\mathbb{H}^{3}$ is cocompact.

\section{A NEGATIVELY CURVED SPACE WHICH IS NOT CONFORMAL AT INFINITY}

All of the constructions of the paper can be carried out in a space of variable negative curvature. However, unless that space is quasi-isometric with a space of constant negative curvature, the corresponding covers at infinity defined by combinatorial disks need not form a conformal sequence. Somehow one must use the group structure associated with an action in order to avoid nonconformality at infinity. We illustrate the difficulty with an explicit example. The space we describe is homogeneous, with varying negative sectional curvature at each point.

Up to quasi-isometry, we have three alternatives for describing our space: as a locally finite graph, as a Riemannian manifold of variable negative sectional curvature, or as a space with a taxicab metric. The combinatorial disks or combinatorial disk-pairs defined by the three methods will be nicely comparable with one another by the methods studied in Section 4. Being comparable with one another, they define comparable combinatorial moduli at infinity by Theorem 4.3.1. Hence conformality at infinity may be studied by means of any of the descriptions. We choose the description by taxicab metric, since it requires the least background and has the most easily discoverable half-spaces.

Consider the three line elements $\left(1 / z^{a}\right) d x,\left(1 / z^{b}\right) d y$, and $(1 / z) d z$, with $a$ and $b$ positive, and use these to construct a taxicab metric $d s$ on upper-half 3 -space

$$
H=\{(x, y, z) \mid z>0\} .
$$

Recall that, in a taxicab metric, one considers only paths that are piecewise parallel to the axes and uses the appropriate line element to measure each component segment. 
8.1. Theorem. The space $H$ with metric $d s$ is a negatively-curved proper metric space whose space at infinity is the 2-sphere $\mathbf{S}^{2}$. If $a \neq b$, the space $H$ is not quasiisometric with hyperbolic 3-space $\mathbb{H}^{3}$; indeed, the disk-pair covers of $\mathbf{S}^{2}$ induced by ds do not form a conformal sequence.

The proof will appear in Subsection 8.3, after preparation.

Remarks. The metric $d s$ is quasi-isometric with the complete, homogeneous Riemannian metric

$$
\left(1 / z^{2 a}\right) d x^{2}+\left(1 / z^{2 b}\right) d y^{2}+\left(1 / z^{2}\right) d z^{2} .
$$

If $a=b=2$, the latter is the hyperbolic metric on $H$. The plane $\{y=0\}$ under the transformation $(x, 0, z) \mapsto\left(a x, z^{a}\right)$ is isometric with scaled hyperbolic space $\{(X, Z) \mid Z>0\}$ having metric $\left(1 / a^{2} Z^{2}\right)\left(d X^{2}+d Z^{2}\right)$. Similarly, the plane $\{x=0\}$ is isometric with a scaled hyperbolic space. That is, the Riemannian space is negatively curved with varying negative curvature. That $(H, d s)$ is negatively curved with $\mathbf{S}^{2}$ at infinity follows easily. However, we shall give an independent proof.

8.2. Geodesics in $(H, d s)$. Let $\alpha \in H$ be any point, and let $P=\left\{P_{1}, \ldots, P_{k}\right\}$ denote any set of axis-parallel vectors. Then there is a path $(\alpha, P)$ in $E^{3}$ which begins at $\alpha$ and follows, successively, the vectors $P_{1}, \ldots, P_{k}$. The terminal endpoint of $(\alpha, P)$ is the vector

$$
\beta=\alpha+P_{1}+\cdots+P_{k} .
$$

If $\beta \in H$, then the set $P$ can be permuted (by a function $\phi$ ) so that the resulting path $(\alpha, \phi(P))$ lies in $H$. Among all paths $(\alpha, \phi(P))$ in $H$, those of smallest $d s$ length are characterized as follows: first list all vectors of $P$ which are parallel to the $z$-axis and rise, then those in the $x$ and $y$ directions (any order), and finally those falling. If two parallel to the $x$-axis have opposite directions, they can be replaced by their vector sum and the path can be shortened, and similarly for the $y$-axis. We may therefore conclude the following.

8.2.1. Theorem. Any geodesic in $H$ must consist of a rising arc, followed by an $(x, y)$-monotone path, followed by a falling arc.

8.2.2. The length of an $(x, y)$-monotone path from $\left(x_{1}, y_{1}, z\right)$ to $\left(x_{2}, y_{2}, z\right)$ is

$$
\frac{1}{z^{a}}\left|x_{2}-x_{1}\right|+\frac{1}{z^{b}}\left|y_{2}-y_{1}\right|
$$

8.2.3. If $\left(x_{1}, y_{1}\right) \neq\left(x_{2}, y_{2}\right)$, then there exists a unique $z \in(0, \infty)$ such that

$$
2=\frac{a}{z^{a}}\left|x_{2}-x_{1}\right|+\frac{b}{z^{b}}\left|y_{2}-y_{1}\right|
$$

We call this value of $z, z^{*}=z^{*}\left(x_{1}, y_{1}, x_{2}, y_{2}\right)$.

Proof of 8.2.3. The right-hand side decreases monotonically from $\infty$ to 0 . 
8.2.4. If $\left(x_{1}, y_{1}, z_{1}\right),\left(x_{2}, y_{2}, z_{2}\right) \in H$ and $\left(x_{1}, y_{1}\right) \neq\left(x_{2}, y_{2}\right)$, then the maximal height attained by a geodesic joining these points is $\max \left\{z_{1}, z_{2}, z^{*}\right\}$, where $z^{*}=$ $z^{*}\left(x_{1}, y_{1}, x_{2}, y_{2}\right)$.

Proof of 8.2.4. We must minimize

$$
f(z)=\ln \left(z / z_{1}\right)+\left(1 / z^{a}\right)\left|x_{2}-x_{1}\right|+\left(1 / z^{b}\right)\left|y_{2}-y_{1}\right|+\ln \left(z / z_{2}\right)
$$

subject to $z \geq z_{1}, z_{2}$. We set the derivative of $f$ equal to 0 and find that

$$
0=2 / z-\left(a / z^{a+1}\right)\left|x_{2}-x_{1}\right|-\left(b / z^{b+1}\right)\left|y_{2}-y_{1}\right|
$$

which defines $z^{*}$. The derivative is negative for $z<z^{*}$ and positive for $z>z *$, so that the result follows.

8.2.5. If $a<b$, then

$$
\frac{2}{b} \leq\left(\frac{\left|x_{2}-x_{1}\right|}{\left(z^{*}\right)^{a}}+\frac{\left|y_{2}-y_{1}\right|}{\left(z^{*}\right)^{b}}\right) \leq \frac{2}{a}
$$

Proof of 8.2.5. Replace the numerator $a$ in the equality of (8.2.3) by $b$, or vice versa. Result (8.2.5) follows.

Summary. A geodesic rises until

$$
\frac{a}{z^{a}}\left|x_{2}-x_{1}\right|+\frac{b}{z^{b}}\left|y_{2}-y_{1}\right| \leq 2,
$$

then follows an $(x, y)$-monotone path, then falls to the terminal endpoint. Note that the length of an $(x, y)$-monotone path has upper bound comparable to 2 .

8.2.6. Corollary. All rays that are not vertically upward are eventually vertically downward.

8.2.7. Corollary. The space $H$ with metric ds is negatively curved.

Proof of 8.2.7. The typical triangle has the form of Figure 8.2.7.

This triangle is made up of three intervals:

$$
\begin{gathered}
{[\alpha, \beta]=\left[\alpha, \alpha^{\prime}, \beta^{\prime \prime}, \beta^{\prime}, \beta\right],} \\
{[\alpha, \gamma]=\left[\alpha, \alpha^{\prime}, \alpha^{\prime \prime}, \gamma^{\prime \prime}, \gamma^{\prime}, \gamma\right],} \\
{[\beta, \gamma]=\left[\beta, \beta^{\prime}, \gamma^{\prime}, \gamma\right] .}
\end{gathered}
$$

Sides $\left[\alpha^{\prime \prime}, \gamma^{\prime \prime}\right],\left[\alpha^{\prime}, \beta^{\prime \prime}\right]$, and $\left[\beta^{\prime}, \gamma^{\prime}\right]$ are all on the order of $2 / a$ in length. Since $\left[\alpha^{\prime}, \beta^{\prime \prime}\right]$ and $\left[\beta^{\prime}, \gamma^{\prime}\right]$ are already small, $\left[\alpha^{\prime \prime}, \gamma^{\prime \prime}\right]$ cannot be much higher than $\left[\alpha^{\prime}, \beta^{\prime \prime}\right]$. Hence the part of $[\alpha, \gamma]$ which is above the level of $\left[\alpha^{\prime}, \beta^{\prime \prime}\right]$ is small. Since $\left[\beta^{\prime}, \gamma^{\prime}\right]$ is small, $\left[\beta^{\prime}, \beta^{\prime \prime}\right]$ and the portions of $\left[\gamma^{\prime}, \gamma^{\prime \prime}\right]$ on the same levels are near one another. It follows that the triangle $\mathcal{P}[\alpha, \beta, \gamma]$ is thin. 


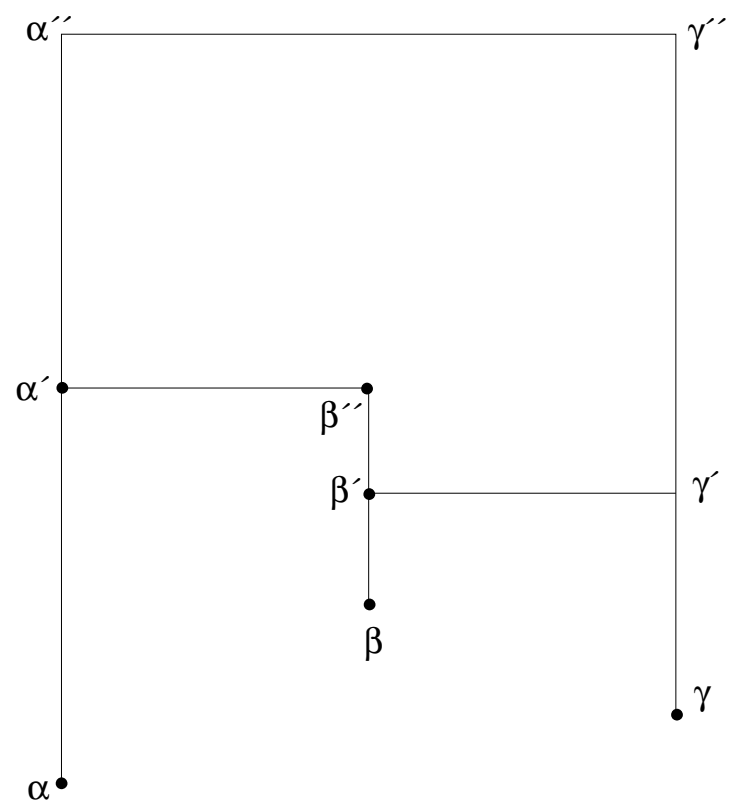

Figure 8.2.7

8.3. Half-spaces. Consider a ray $R$. We know by (8.2.6) that we may assume $R$ is vertically downward, say equal to the portion of the $z$-axis below $(0,0,1)$. The typical projection arc to $R$ will travel vertically upward until the $(x, y)$-monotone distance to $R$ is on the order of 2 (see (8.2.5)), then it will travel horizontally to $R$. Thus, in order to see the half space $H(R, n)$, we need to see the 2-neighborhood of $R$ in the plane $\ln (1 / z)=n$. More precisely, we need the neighborhood defined by the inequality

$$
a \cdot e^{n a} \cdot|x|+b \cdot e^{n b} \cdot|y| \leq 2,
$$

which defines a diamond shaped box in the $(x, y)$-plane. This box, in the first quadrant, is bounded by the line

$$
y=\frac{2-a \cdot e^{n a} \cdot x}{b \cdot e^{n b}}
$$

which has slope

$$
-\frac{a}{b \cdot e^{n(b-a)}}
$$

Assuming $b>a$, we find that the box becomes flatter and flatter as $n \rightarrow \infty$.

Proof of Theorem 8.1. Since the combinatorial disks become relatively skinny (flattened) as they become small, it is an easy matter to show that the combinatorial modulus of a Euclidean rectangle defined by such combinatorial disks approaches either 0 or $\infty$. This contradicts the axioms of conformal sequence. We conclude that $H$ with metric $d s$ cannot be quasi-isometric with hyperbolic 3 -space $\mathbb{H}^{3}$. 


\section{REFERENCES}

1. Alonso, J. M.; Brady, T.; Cooper, D.; Ferlini, V.; Lustig, M.; Mihalik, M.; Shapiro, M.; and Short, H., Notes on word hyperbolic groups, Group theory from a geometric viewpoint (E. Ghys, A. Häfliger, and A. Verjovsky, eds.), World Scientific, Singapore, 1991. MR 93a:20001

2. Ballmann, W.; Ghys, E.; Haefliger, A.; de la Harpe, P.; Salem, E.; Strebel, R.; and Troyanov, M., Sur les groupes hyperboliques d'après Mikhael Gromov, available from the authors (The "little green book."), 1989.

3. Beardon, A. F., The geometry of discrete groups, Discrete Groups and Automorphic Functions (W. J. Harvey, eds.), Academic Press London-New York-San Francisco, 1977, pp. 47-72. MR $\mathbf{5 7 : 1 3 6 7 0}$

4. Bowditch, B. H., Notes on Gromov's hyperbolicity criterion, Group theory from a geometric viewpoint, World Scientific, Singapore (to appear).

5. Cannon, J. W., The combinatorial structure of cocompact discrete hyperbolic groups, Geom. Dedicata 16 (1984), 123-148. MR 86j:20032

6. - The theory of negatively curved spaces and groups, Ergodic Theory, Symbolic Dynamics and Hyperbolic Spaces (Tim Bedford, Michael Keane and Caroline Series, eds.), Oxford Univ. Press, Oxford-New York-Toronto, 1991, pp. 315-369. MR 93e:58002

7. _ The combinatorial Riemann mapping theorem, Acta Math. 173 (1994), 155-234. MR 95k:30046

8. Cannon, J. W., and Cooper, Daryl, A characterization of cocompact hyperbolic and finitevolume hyperbolic groups in dimension three, Trans. Amer. Math. Soc. 330 (1992), 419-431. MR 92f: 22017

9. Cannon, J. W.; Floyd, W. J.; and Parry, Walter, Squaring rectangles: the finite Riemann mapping theorem, The Mathematical Heritage of Wilhelm Magnus - Groups, Geometry \& Special Functions, Contemp. Math., vol. 169, Amer. Math. Soc., Providence, RI, 1994, pp. 133-212. MR 95g:20045

10. Coornaert, M.; Delzant, T.; and Papadopoulos, A., Géométrie et théorie des groupes. Les groupes hyperboliques de Gromov, Lecture Notes in Math. 1441, Springer-Verlag, BerlinHeidelberg-New York, 1990. MR 92f:57003

11. Epstein, D. B. A.; Cannon, J. W.; Holt, D. F.; Levy, S. V. F.; Paterson, M. S.; and Thurston, W. P., Word Processing in Groups, Jones and Bartlett, Boston-London, 1992. MR 93i:20036

12. Floyd, W. J., Group completions and limit sets of Kleinian groups, Inv. Math. 57 (1980), 205-218. MR 81e:57002

13. Furstenberg, H., A Poisson formula for semi-simple Lie groups, Ann. of Math. 77 (1963), 335-386. MR 26:3820

14. _ Boundaries of Lie groups and discrete subgroups, Actes, Congrès Intern. Math. (Nice, 1970), vol. 2, pp. 301-306. MR 55:3167

15. Gromov, M., Hyperbolic groups, Essays in group theory (S. M. Gersten, ed.), MSRI Publ. 8, Springer-Verlag, Berlin-Heidelberg-New York, 1987, pp. 75-263. MR 89e:20070

16. Gromov, M., and Thurston, W., Pinching constants for hyperbolic manifolds, Inv. Math. 89 (1987), 1-12. MR 88e:53058

17. Kuusalo, Tapani, Verallgemeinerter Riemannscher Abbildungssatz und quasikonforme Mannigfaltigkeiten, Ann. Acad. Sci. Fenn. Ser. A I 409 (1967), 24 pages. MR 36:1645

18. Lehner, Joseph, Discontinuous Groups and Automorphic Functions, Amer. Math. Soc., Providence, R. I. (1964). MR 29:1332

19. Lehto, O., and Virtanen, K. I., Quasiconformal Mappings in the Plane, Springer-Verlag, Berlin-Heidelberg-New York, 1973. MR 49:9202

20. Martin, G. J., Discrete quasiconformal groups that are not the quasiconformal conjugates of Möbius groups, Ann. Acad. Sci. Fenn. Ser. A I 11 (1986), 179-202. MR 89d:30025

21. Maskit, B., Kleinian Groups, Springer-Verlag, Berlin-Heidelberg-New York, 1988. MR 90a:30132

22. Mostow, G. D., Strong Rigidity of Locally Symmetric Spaces, Princeton Univ. Press, Princeton, N. J., 1973. MR 52:5874

23. Mostow, G. D., and Siu, Y. -T., A compact Kähler surface of negative curvature not covered by a ball, Ann. Math 112 (1980), 321-360. MR 82f:53075

24. Rüedy, Reto A., Deformations of embedded Riemann surfaces, Advances in the Theory of Riemann Surfaces (Ahlfors, Bers, et al., eds.), Princeton Univ. Press, Princeton, N. J., 1971, pp. 385-392. MR 44:7600 
25. Siegel, C. L., Topics in Complex Function Theory, vol. II, Wiley-Interscience, New YorkLondon-Sydney-Toronto, 1971.

26. Sullivan, Dennis, On the ergodic theory at infinity of an arbitrary discrete group of hyperbolic motions, Riemann Surfaces and Related Topics: Proceedings of the 1978 Stony Brook Conference (Irwin Kra and Bernard Maskit, eds.), Princeton University Press, Princeton, N.J., 1981, pp. 465-496. MR 83f:58052

27. Swenson, Eric Lewis, Negatively curved groups and related topics, Ph.D. Dissertation, Brigham Young Univ, 1993.

28. Thurston, W. P., Three dimensional manifolds, Kleinian groups and hyperbolic geometry, Bull. Amer. Math. Soc. 6 (1982), 357-381. MR 83h:57019

29. Tukia, P., A quasiconformal group not isomorphic to a Möbius group, Ann. Acad. Sci. Fenn. Ser. A I Math. 6 (1981), 149-160. MR 83b:30019

30. Tukia, Pekka, On quasiconformal groups, J. Analyse Math. 46 (1986), 318-346. MR 87 m:30043

Department of Mathematics, Brigham Young University, Provo, Utah 84602

E-mail address: cannon@math.byu.edu

E-mail address: eric@math.byu.edu 